\title{
(IM)POSIBILIDADES DE LA VIVIENDA PARTICIPATIVA: RETORNANDO AL SISTEMA FLEXIBO
}

\author{
(IM)POSSIBILITIES OF PARTICIPATORY HOUSING: REVISITING THE FLEXIBO SYSTEM
}

Rodrigo Rieiro Díaz, Kim Haugbølle

RESUMEN El objetivo de este texto es analizar qué puede aprender la arquitectura contemporánea de las experiencias históricas de la arquitectura participativa. Partiendo de la teoría de la construcción social de la tecnología (SCOT), se estudia la tradición de vivienda participativa de los años sesenta y setenta. Se identifican los problemas a los que esta se enfrentó y cómo terminó resultando marginal. El artículo se centra en el caso ejemplar de Flexibo, un sistema de vivienda de soportes y aportes desarrollado en Dinamarca en los años setenta. Ciertas condiciones sociales e históricas en los países nórdicos establecieron un caldo de cultivo óptimo para la democratización de la vivienda, por lo que en estos países la influencia de la arquitectura participativa, si bien discreta, ha sido amplia. No obstante, el estudio revela la principal limitación de la arquitectura participativa tal como ésta sucedió: la mayor parte de los esfuerzos hacia la democratización de la vida cotidiana por parte de la arquitectura no fueron acompañado por la democratización de los procesos y las prácticas arquitectónicas. Ciertas experiencias coetáneas manifiestamente alternativas, se apuntan finalmente como pistas para aquellos interesados hoy en alcanzar el viejo objetivo de una participación transformadora. PALABRAS CLAVE participación; Flexibo; Dinamarca; democracia vecinal; soportes y aportes; SCOT

SUMMARY The purpose of this paper is to discuss what contemporary architecture can learn from historical experience of participatory architecture. Based on the theory of social construction of technology (SCOT), this paper analyses the tradition of participatory architecture from the 1960s and 1970s. It identifies the problems faced by participatory architecture, and how participatory architecture turned out to be marginal to mainstream architecture. This paper focuses on the exemplary case of Flexibo, a housing system of support and infill developed in Denmark in the 1970s. Certain socio-historical conditions in the Nordic countries presented an optimal breeding ground for housing democracy, and in these countries the influence of participatory architecture, though modest, is still broad. Nevertheless, the study reveals the main limitation of this participatory architecture as it emerged: most of the efforts towards democratisation of everyday life by architecture came unaccompanied by parallel efforts towards democratisation of the everyday of architectural processes and practices. Finally, this paper points out some alternative ways to overcome the previous missteps for those today who are still interested in the old goal of a transforming participatory architecture.

KEY WORDS participation; Flexibo; Denmark; tenant's democracy; support + infill; SCOT

Persona de contacto / Corresponding author: rodrigo_rieiro@hotmail.com. Escuela Técnica Superior de Arquitectura, Universidad Politécnica de Madrid. España 


\section{INTRODUCCIÓN}

a vivienda, así como la arquitectura en general, ha sido a menudo presentada como un asunto pospolítico, es decir, más allá de los mecanismos tradicionales de organización de la esfera pública. Para muchos, estaría técnica o económicamente determinada, en particular durante las pasadas décadas de retirada de la dimensión política del discurso dominante en arquitectura.

Otros han abordado la vivienda como una responsabilidad política. El papel de los arquitectos en la mejora de la sociedad a través de un proceso de reorientación de nuestros sistemas tecnológicos inspirado por principios democráticos ha sido una preocupación recurrente de la disciplina y la profesión arquitectónicas, manifiestamente desde cierta tradición del Movimiento Moderno, que se opone a la rigidez y el autoritarismo del diseño total y la Gesamtkunstwerk ${ }^{1}$ y encuentra expresión en la concepción moderna del espacio, igual y neutro, como un escenario dispuesto para que la vida suceda.

Ha habido una corriente minoritaria pero recurrente de arquitectos animada por una parte del público en muchos países dispuesta a incorporar a habitantes y profesionales en la concepción de vivienda. Esta corriente avanzó a la vanguardia del debate arquitectónico en los años sesenta y setenta de la mano de las ideas de participación ciudadana. Sin embargo, el éxito de la empresa ha sido limitado por ahora y la democratización de los procesos de alojamiento humano sigue siendo una tarea pendiente.

Este es el telón de fondo para una nueva generación de arquitectos que estaría renovando y repensando esas controversias, apoyada por un creciente interés en modelos de vivienda alternativos por parte del público en el contexto posterior a la crisis financiera de 2008 y al estallido de la burbuja inmobiliaria.

Este artículo repasa la tradición de la arquitectura participativa de los años sesenta y setenta, centrándose en las condiciones socio-históricas especialmente idóneas que ésta encontró en los países nórdicos. Se sostiene que un caso ejemplar de esta corriente, Flexibo en Copenhague, encarna el defecto crucial de la arquitectura participativa: irónicamente, trató de implementar vivienda democrática a través de roles y procesos autoritarios.

Otras experiencias interesantes en este campo en los países nórdicos, hasta ahora poco conocidas en círculos académicos anglófonos o hispanohablantes, apuntan a una arquitectura de participación del siglo XXI destinada a elevar la calidad media de la práctica cotidiana de la edificación en beneficio de todos ¿Podrían estos 
experimentos nórdicos marginales ayudar a esta generación del siglo XXI a alcanzar el renovado objetivo de una vivienda democrática??

\section{CONSIDERACIONES METODOLÓGICAS \\ Teoría}

La arquitectura participativa, tal como se desarrolló históricamente, implica una caracterización del conocimiento arquitectónico como de tipo fronético ${ }^{3}$, es decir, como una sabiduría práctica, subida a los hombros del conocimiento científico y técnico, y dirigida hacia una buena vida.

A su vez, esto implica una comprensión de las obras arquitectónicas como constructos tecno-políticos. Se ha considerado que el constructivismo social de la tecnología (SCOT) ofrece un andamiaje útil para estudiar el surgimiento y la evolución de dichos constructos. De hecho, la idoneidad de esta metodología para el estudio de la política de la arquitectura ha sido mantenida por algunos de sus principales defensores ${ }^{4}$.

Algunas voces críticas han alertado también sobre las limitaciones del reduccionismo inherente a esta teoría. Este texto aspira a una comprensión más amplia y profunda de la dinámica evidente en las obras arquitectónicas. Por lo tanto, una cierta posición materialista no determinista subyace en el mismo, dentro de la diversa tradición en la cual superestructura y base se determinan e interrelacionan mutuamente.

La tradición nórdica de diseño participativo ofrece fundamentos teóricos para la congruencia entre este compromiso, el conocimiento fronético y la cocreación ${ }^{5}$. Por otra parte, se ha considerado tan necesario como coherente con el objeto de estudio una valoración de lo que significan los casos estudiados en términos de calidad de la vida cotidiana, distribución del poder en la sociedad y libertad colectiva.

\section{Ámbito de estudio}

En primer lugar, el ámbito del estudio se ha limitado a la vivienda. La vivienda ha sido el tema principal de la arquitectura, al menos tal como profesión y disciplina establecieron a partir del Movimiento Moderno. También fue el principal motivo de preocupación para los arquitectos y el público comprometido con la participación en la arquitectura en los años sesenta y setenta. Simultáneamente, existe una alta prevalencia de viviendas no diseñadas por arquitectos, y por razones muy diferentes. Por último, pero no menos importante, plantea problemas técnicamente complejos a partir de una escala muy pequeña, justificando la utilidad de un profesional en un asunto en el que los no profesionales suelen tener un amplio conocimiento práctico.

La vivienda participativa sigue siendo un concepto bastante vago. La directriz para restringir el campo de estudio ha sido reducirlo a los casos en los que los sujetos de la acción constructiva fueron colectivos no exclusivos que integraran profesionales y no profesionales. Probablemente, siempre hay algún nivel de participación en la toma de decisiones en todos los esfuerzos humanos de tanta complejidad como la vivienda. Por lo tanto, tiene poco sentido hablar en términos binarios (de procesos participativos o no participativos). El foco se ha dirigido hacia experiencias que se sitúan arriba de la canónica "escala de la

2. Por democracia aquí no se entiende un bien en sí mismo, sino, en palabras de Simone Weil apoyándose en Rousseau, un medio hacia un buen fin: "no que algo sea correcto porque a la gente le gusta, sino que, bajo ciertas condiciones, la voluntad popular es más probable de estar de acuerdo con la justicia que cualquier otra".WEIL, Simone. Nota sobre la supresión de los partidos políticos. En: WEIL, Simone; VALLS, Sylvia María (ed.). Profesión de fe. Antología y crítica alrededor de su obra. Versión corregida y aumentada. México: Pleroma, 2007, pp. 61-73 (62-63). Weil añade: "la simple afirmación de estas condiciones muestra que nunca hemos conocido nada remotamente parecido a una democracia". Ibid., p. 64. Se ha realizado la traducción de todas las citas por parte de los autores.

3. Este término de origen aristotélico se usa aquí en el sentido que toma en su adaptación al discurso arquitectónico por Inge Mette Kirkeby y Bent Flyvberg: KIRKEBY, Inge Mette. Knowledge in the making. Architectural Research Quarterly. Cardiff: Welsh School of Architecture, 2009, vol. 13, n 3/4, pp. 307-13. FLYVBJERG, Bent. Phronetic Planning Research: Theoretical and Methodological Reflections. Planning Theory and Practice, Routledge, 2004, vol. 5, n $n^{\circ}$, pp. 283-306.

4. Por ejemplo para el estudio del urbanismo de Barcelona. AIBAR, Eduardo; BIJKER, Wiebe E. Constructing the city: The Cerdá Plan for the extension of BarceIona. Science, Technology and Human Values, Sage Pub., enero 1997, vol. 22, nº 1, pp. 3-30.

5. Cocreación aquí alude genéricamente a cualquier acto de creación colectiva. 


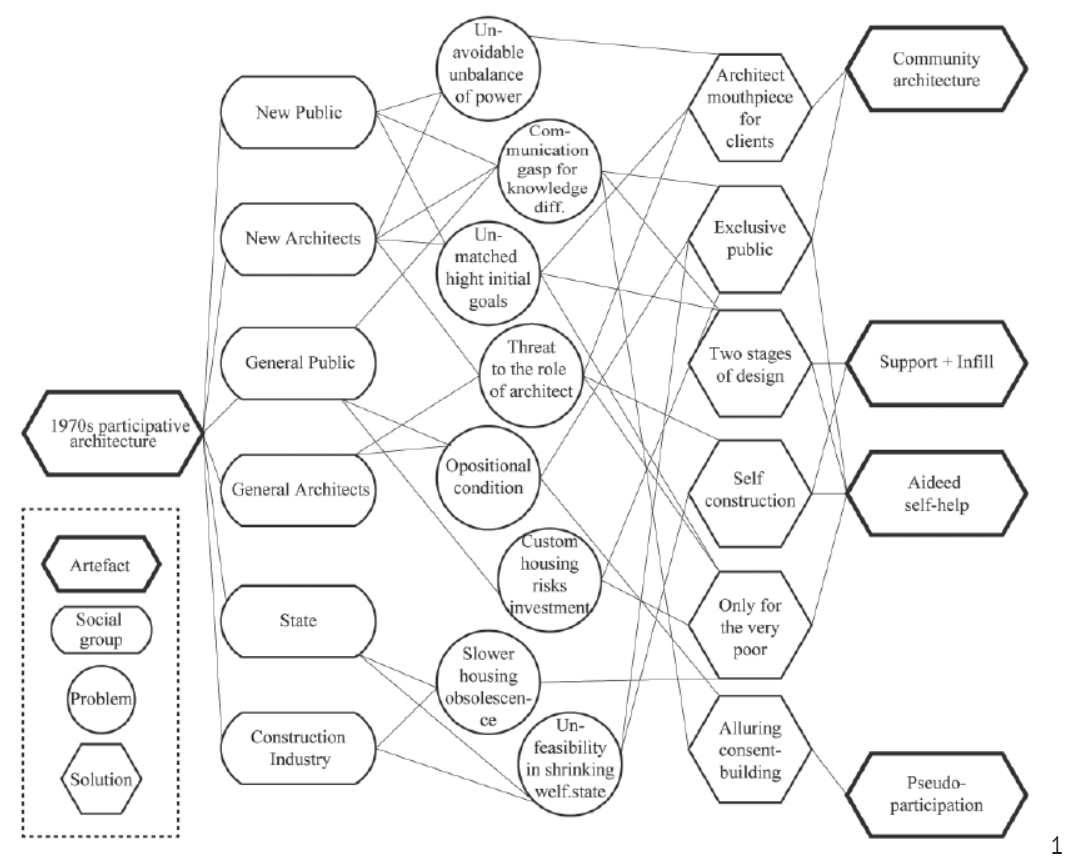

participación ciudadana" de Arnstein, ${ }^{6}$ dejando de lado los casos en los que la participación no estaba acompañada de un alto nivel en la toma de decisiones.

\section{Métodos}

Este artículo es principalmente el resultado de una revisión crítica de la literatura disponible sobre la tradición de la vivienda participativa desde la década de 1960 hasta la actualidad ${ }^{7}$. Existe una orgullosa historia de participación ciudadana en los países nórdicos, por lo que existe una tradición de fomento de la participación en muchos campos, incluidos los relacionados con la vivienda.

A continuación este texto se centra en el estudio de un destacado ejemplo de arquitectura participativa en vivienda. El proyecto de vivienda Flexibo es un caso ejemplar de esta tradición de vivienda participativa, pero al mismo tiempo apunta algunos de sus fallos, limitaciones y dilemas. La información para su estudio fue obtenida de publicaciones arquitectónicas locales de los años 70 y 80 , visitas a los edificios y documentación técnica. Se accedió a esta última a través del archivo físico del Centro de Edificios (Bygninger Center) del municipio de Copenhague.
VIVIENDA PARTICIPATIVA: EMERGENCIA Y PRINCIPIOS La emergencia y los principios de la vivienda participativa forman parte de la historia de la democratización de la arquitectura, la vida cotidiana, el hogar, el trabajo y el vecindario. La siguiente sección repasa brevemente el desarrollo de las tres primeras áreas, que forman parte del telón de fondo que explica el surgimiento de la vivienda participativa en los años sesenta.

La figura 1 aporta una visión general de los grupos sociales más relevantes, los principales problemas y soluciones, así como los artefactos resultantes, en relación a la vivienda participativa de los años setenta. Un grupo social relevante formado por parte del público y algunos arquitectos interesados en la arquitectura participativa identificaron un conjunto de problemas, como el desequilibrio de poder. El grupo mayoritario del público y los arquitectos convencionales, junto al estado o la industria de la construcción, afrontaron otros, como la inviabilidad económica de la empresa en el contexto de un estado de bienestar en retroceso. Los soluciones experimentadas condujeron a nuevos artefactos tecnológicos en los que o bien predominaba un público exclusivo, o bien la participación era muy baja, o bien arquitecto y usuario se sucedían en el planeamiento de la vivienda (figura 1). 


\section{Democracia en la arquitectura}

Desde finales de los años sesenta, se fue haciendo cada vez más popular en la cultura arquitectónica de las naciones del primer mundo cuestionar que la vivienda de la clase trabajadora estaba planeada para el trabajador, no por el trabajador. Como se plantea en el seminal manifiesto de Giancarlo de Carlo El Público de la Arquitectura ${ }^{8}$, el proyecto de una arquitectura de participación" "liberadora y democrática" no sólo debía dar legitimidad política a la planificación, sino transformar radicalmente la disciplina y la profesión. Implicaría ir más allá del cómo, donde los arquitectos habían encontrado tradicionalmente su sustento, para retomar el porqué y el qué hacer en arquitectura. En términos aristotélicos se trataba de ir más allá del techne -el saber hacer técnico-y el episteme -conocimiento científico- para traer de vuelta a la disciplina arquitectónica la phronesis -sabiduría práctica que se apoya en los otros dos-.

A lo largo y alrededor de la década de 1970 se realizaron varios proyectos de viviendas conjuntamente por sus habitantes y arquitectos. Nuovo Villaggio Matteotti, de Giancarlo de Carlo, Byker Wall, de Ralph Erskine, las viviendas de la Facultad de Medicina de Lovaina, de Lucien Kroll, la Ökohaus de Frei Otto en Berlín y las casas autoconstruidas en Lewisham, de Walter Segal, son probablemente los ejemplos de vivienda participativa más publicados en los medios arquitectónicos.

Margaret Crawford expuso cómo las reclamaciones de los arquitectos radicales eran en realidad una negación incompleta, simplemente invirtiendo los papeles ya ficticios del arquitecto todopoderoso y del cliente ideal, las masas, pero aceptando los supuestos ideológicos en los que se apoyaban. Frente a su ineficacia para efectuar cambios sociales, éstos se fueron centrando en la mera oposición a la estética moderna para acabar "reemplazando al estado de bienestar del movimiento moderno por un mercado en el que su cliente ideal no tenía los medios para adquirir servicios arquitectónicos"10.

La falta de agencia social ${ }^{11}$ conllevó una estetización de lo político. Tahl Kaminer ha desarrollado en Architecture, crisis, and resuscitation cómo el fracaso de algunos posicionamientos críticos de la arquitectura de los años setenta condujo entonces a una retirada a la autonomía de la arquitectura de papel y de ahí al panorama actual de arquitectos estrella ${ }^{12}$.

Lo político en vivienda evolucionó también hacia el espectáculo. Por un lado, algunos vieron la participación como un medio para construir consenso, promoviendo la sensación de haber influido en las decisiones, y afectando positivamente en la aceptación general de los resultados. Esta seudo participación terminó por dificultar aún más la participación auténtica en algunos contextos ${ }^{13}$.

La dimensión política del diseño participativo de vivienda también fue agotada por la invocación al mito de la comunidad uniforme. En la community architecture ${ }^{14}$, tanto la autoridad del arquitecto como la de los ciudadanos se disuelven en nombre del mito de una comunidad purificada, "definida por grupos socialmente homogéneos,

8. DE CARLO, Giancarlo. Architecture's public. En: BLUNDELL JONES, Peter; PETRESCU, Doina; TILL, Jeremy, eds. Architecture and participation. Abingdon: Spon Press, 2005, pp. 3-22 (p. 15).

9. Durante los 60, la palabra participación se convirtió en parte del vocabulario político popular, especialmente entre los estudiantes. En este contexto, la participación se refiere a la democracia directa, en oposición al ethos de la democracia liberal, así como un redescubrimiento de la teoría democrática tradicional, incluyendo las visiones de Rousseau y Aristóteles.

10. CRAWFORD, Margaret. Can architects be socially responsible? En: GHIRARDO, Diane, ed.. Out of site: a social criticism of architecture. Seattle: Bay Pres, 1991, pp. 27-45 (p. 39).

11. "En el ámbito de la filosofía y la sociología, se denomina agencia a la capacidad que posee un agente (una persona u otra identidad) para actuar en un mundo." https://es.wikipedia.org/wiki/Agencia_(filosof\%C3\%ADa)

12. KAMINER, Tahl. Architecture, crisis and resuscitation. New York: Routledge, 2011.

13.QUERRAIN, Anne. How inhabitants can become collective developers: France 1968-2000. En: BLUNDELL JONES, Peter; PETRESCU, Doina; TILL, Jeremy, eds. Architecture and participation. Abingdon: Spon Press, 2005, pp. 105-15 (p. 113). ELLIN, Nan. Participatory Architecture of the Parisian periphery: Lucien Kroll's Vignes Blanches. Journal of Architectural Education, Routledge, primavera 2000, vol. 53, nº 3, pp. 178-83 (p. 181)..

14. Movimiento arquitectónico originario del Reino Unido que abogó principalmente en los años 70 y 80 por el involucramiento del usuario en el diseño, la construcción y la administración del entorno físico. 
en el peor de los casos impulsada por el propio interés, y por lo tanto exclusiva"15.

Las prácticas de aquella generación de arquitectos comprometidos con la participación les llevaron a la ineficiencia social. La "profunda reformulación tanto de la teoría como de la práctica para evitar repetir las estrategias bienintencionadas pero erróneas utilizadas por los reformistas modernos y los radicales de los sesenta"16 sigue siendo una tarea tan pendiente como cuando Crawford la formuló hace veinticinco años. El paisaje espectacular de los desarrollos urbanos contemporáneos, donde los edificios icónicos complementan la variación superficial de las fachadas de viviendas por otro lado repetitivas, representa bien el ethos de la democracia liberal al que los no conformistas se habían opuesto.

\section{Democracia en lo cotidiano}

La relación entre la arquitectura y lo cotidiano ${ }^{17}$ clarifica si la vivienda participativa puede llegar a ser una herramienta política para un proyecto de emancipación. Precisamente a través de un compromiso personal con lo cotidiano, parte de aquella generación esperaba que se podrían alcanzar utopías prácticas. Una cotidianidad desnaturalizada exigiría juicio. Es en la forma en que todos y cada uno tenemos una relación consciente con lo cotidiano como, siguiendo la máxima feminista, lo personal se convierte en político. En consecuencia, el tipo de conocimiento práctico debería implicar un enfoque hacia la vida cotidiana, la gente normal y lo común en general. La renuncia a lo cotidiano ha sido entendida por algunos arquitectos, especialmente en los años setenta, como una derogación de las responsabilidades profesionales.
La creciente demanda de libertad, individualismo y creatividad por parte de la sociedad posfordista fomentó la adopción de una crítica artística de la sociedad mientras que la impotencia política llevó a una retirada de la crítica social. Algunos arquitectos encontraron en la arquitectura cotidiana realizada por no profesionales espacios en los que la diferencia, la alteridad y lo otro podían florecer.

En aquella época, el atractivo de la edificación "sin pedigrí" fue subrayado por el éxito de la exposición y el libro de Rudofsky Arquitectura sin arquitectos. Blundell Jones observa cómo en el popular libro de Boudon sobre las viviendas de Le Corbusier en Pessac ${ }^{18}$ las modificaciones de los propietarios fueron tomadas tan en serio como para que el autor se atreviese a comparar la expresión de la gente con la del maestro. La espontaneidad, invención y creatividad evidenciadas en este tipo de trabajos de bricolage eran estéticamente atractivos como antídoto contra la obra industrializada de un racionalismo impopular, realizada por los arquitectos del establishment.

No obstante, existe una diferencia fundamental entre la libertad de organización colectiva y la liberación del individuo de las restricciones sociales. Bookchin ha explicado el gran abismo que existe entre el compromiso personalista con la autonomía individual y el compromiso colectivo con la libertad social, remontándolo a la oposición entre el origen de las palabras inglesas liberty, de la libertas romana, donde el ego sin trabas es "libre de poseer su propiedad personal" y freedom, de origen germánico, el dominio de los libres, "donde la individualidad no se opone o separa del colectivo, sino que está formada de forma significativa por su propia existencia social" 19 . No fue la exigencia de la libertad colectiva per se, sino su

15.HARVEY, David. The New Urbanism and the communitarian trap. En: Harvard Design Magazine, [en línea]. Cambridge (MA): Harvard University Graduate School of Design, 1997. n 1 [consulta: 19-03-2018].

TZONNIS, Alexander. Community in the mind. A model for personal and collaborative design. CAADRIA 2000 Proceedings of the Fifth Conference on Computer Aided Architectural Design Research in Asia, Singapore, 18-19 May 2000, pp. 1-14 (p. 3).

16.CRAWFORD, Margaret, op. cit., p. 43.

17. Lo cotidiano alude aquí al término teórico asociado con los escritos de Marx sobre la alienación, central para autores anarquistas y estudiosos marxistas muy influyentes en la época, como Colin Ward, Georg Lúkacs, Henri Lefebvre o Michel de Certeau.

18. BLUNDELL JONES, Peter. Sixty-eight and after. En: BLUNDELL JONES, Peter; PETRESCU, Doina; TILL, Jeremy, eds. Architecture and participation. Abingdon: Spon Press, 2005, pp. 127-40 (p. 134).

19. BOOKCHIN, Murray. Social anarchism or lifestyle anarchism. An unbridgeable chasm. Edinburgh: AK Press, 1995, p. 12-13. 
evolución hacia luchas por la autonomía individual, lo que explica la retirada a una crítica meramente estética.

\section{Democracia en el hogar}

Algunos arquitectos han visto la solución a las muchas dificultades de la arquitectura participativa en la creación de una especie de arquitectura de doble escala, manteniendo esquemas autoritarios en las fases de concepción y construcción, donde tradicionalmente el rol del arquitecto es predominante, y propiciando altos niveles de decisión para los residentes durante el resto del ciclo de vida del edificio, cuando la presencia de los arquitectos ya suele ser menor.

La idea fue claramente articulada por la obra de 1961 de Habraken Soportes: una alternativa a la vivienda de masas ${ }^{20}$. Habraken estableció un sistema en el que la estructura de soporte, tanto como dispositivo técnico como marco social, se diferenciaba de los aportes o acondicionamiento interior en la construcción y proyecto residencial, dando lugar a sus ideas de participación de los usuarios. Esta arquitectura de doble escala también está de acuerdo con una concepción indetermeninada del espacio con muchos precedentes en la historia del Movimiento Moderno ${ }^{21}$.

Esta pista puede seguirse también hacia adelante hasta los acabados en bruto de las viviendas de Lacaton y Vassal o a la premiada vivienda Quinta Monroy, de Elemental y Alejandro Aravena. En este proyecto de vivienda social, los arquitectos diseñan y los profesionales construyen un refugio básico, con financiación pública, a completar por los propios habitantes. Estando la empresa participativa destinada principalmente a transformar la vivienda financiada por el estado del bienestar, no es una gran sorpresa que girase la vista hacia el otro gran sistema de vivienda institucionalmente financiado que existe en el mundo: es imposible ignorar la afinidad entre el sistema de soportes más aportes y los programas precedentes de autoayuda asistida. ${ }^{22}$

\section{REVISITANDO EL CASO EJEMPLAR:}

\section{EL SISTEMA FLEXIBO}

Antes de entrar en el estudio de Flexibo, es importante arrojar luz brevemente sobre dos conceptos, la democracia vecinal y la democracia laboral, que presentan significativas peculiaridades en los países nórdicos, lo que ayuda a entender el surgimiento de este sistema en Dinamarca como un caso ejemplar de vivienda participativa.

\section{Democracia vecinal}

Algunas particularidades del sistema danés de vivienda permitieron prácticas altamente democráticas de alojamiento por parte de colectivos no exclusivos de arquitectos e inquilinos. Para empezar, la línea de trabajo de las sociedades filantrópicas y las organizaciones de ayuda mutua obrera surgida en el siglo XIX nunca fue completamente cooptada por el Estado. Ésta herencia se enmarca en el contexto de una cultura de convivencia, colaboración y trabajo horizontal que ha formado parte de Dinamarca al menos desde la influencia del movimiento cooperativo danés (en danés: andelsbevæge/sen) y de la pedagogía de Grundtvig durante el siglo XIX²3 (figura 2).

El movimiento cooperativo danés, con desarrollos paralelos en los otros países nórdicos, fue principalmente un movimiento campesino y religioso que transformó el medio rural. Su versión urbana, vinculada al ascenso del movimiento obrero internacional, alcanzó un gran impacto en el sector de la vivienda a través de las cooperativas de vivienda (andelsbolig) que, junto con la también autogestionada vivienda social (almen bolig), representan hoy un $26 \%$ de la vivienda danesa, 37\% en Copenhague (figura 3).

La beboerdemokrati (democracia vecinal) es también un legado de la tradición cooperativa de la vivienda

20. HABRAKEN, Nicholas J. Supports: an alternative to mass housing. London: Urban International Press, 1999.

21. SCHNEIDER, Tatjiana; TILL, Jeremy. Flexible housing. Oxford: Architectural Press, 2007.

22. Por autoayuda asistida en vivienda se entiende vivienda construida con ayuda estatal por familias para su propio uso. Para una historia de estos modelos: HARRIS, Richard. Slipping Through the Cracks: The Origins of Aided Self-help Housing, 1918-53. En: Housing Studies, Londres: Carfax Publishing Ltd. 1999, vol. 14, n 3, pp. 281-309.

23. MANNICHE, Peter, Living democracy in Denmark: independent farmers, farmer's cooperation, the folk high schools, cooperation in towns, social and cultural activities, social legislation, a Danish village. 2. ed. Copenhagen: G.E.C. Gad Pub., 1970. 
C. Central cooperative commitee

1. Fed. of Danish coop. bacon factories

2. The Danish farmers' coop. egg-export assoc.

3. Assoc. of Danish coop. cattle export societies

5. Fed. of Danish coop. butter export assoc.

5. Fed. of Danish coop. butter
6. Danish coop. fertilizer soc.

6. Danish coop. fertilizer soc.
7. Danish farmers' coop. assoc. for seed growing

8. Fed. of Danish dairy assoc.

8. Fed. of Danish dairy assoc.
9. The Danish coop. cement factory

10. Coop, coal-society

11. Assoc, of Danish agricultural coop. insurance soc.

12. The sanatorium soc. of the coop. societies

13. Coop. wholesale soc. of Denmark

14. Coop. bank

15. Danish village banks

I. Local dairy assoc.

II. Provincial dairy assoc.

III. Butter export soc.

IV. Coop. insurance soc.

a. Bacon factory

b. Egg-collecting soc.

d. Local assoc. for feeding-stuffs

e. Coop. dairy

e. Coop. dairy
f. Local soc. for fertilizer

g. Coop. distributive store

h. Village bank

M. Individual member
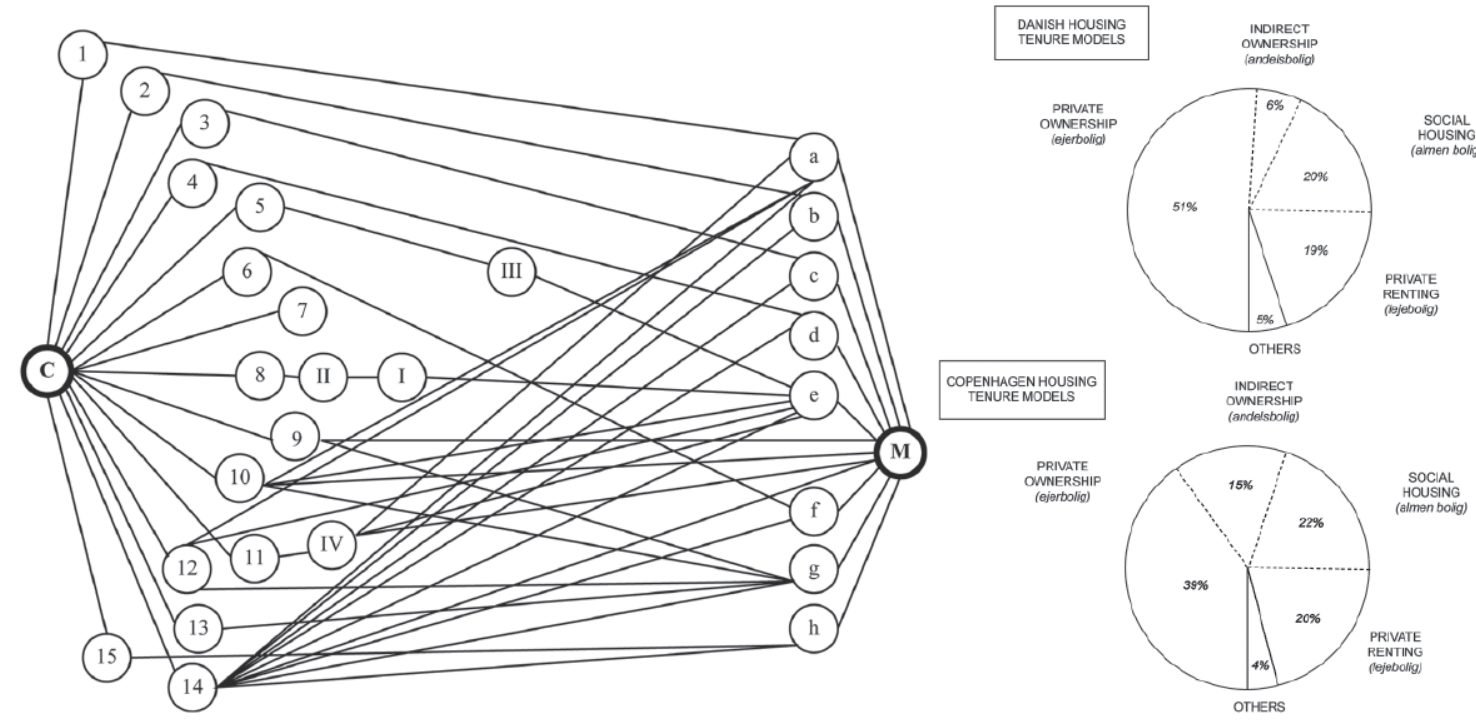

2 danesa del siglo XIX, caracterizada por altos niveles de control de los inquilinos a la escala de inmueble. Con la Ley de Provisión de Vivienda de 1970 los inquilinos fueron autorizados a administrar sus propiedades por medio de juntas de inquilinos democráticamente elegidas. En 1984, se confirma esta tendencia como característica principal del sistema de vivienda social danés con la Ley de Democracia de Inquilinos. Como consecuencia 22.000 inquilinos (de un millón) tienen un puesto electo en las juntas, el 33\% participa en las reuniones anuales de las asociaciones y los inquilinos realizan un $10 \%$ del trabajo de mantenimiento durante los días de trabajo común²4.

\section{Democracia industrial}

El principio de involucrar a los usuarios en los procesos de diseño se ha articulado más claramente fuera de la disciplina arquitectónica por el campo del diseño participativo, que surgió también entre los diversos movimientos sociales y políticos de los años sesenta y setenta, cuando una parte de la sociedad exigió una mayor capacidad en la toma de decisiones sobre diferentes aspectos de su vida.
El diseño participativo hunde sus raíces en los movimientos por la democratización de los lugares de trabajo en los países nórdicos, especialmente en Suecia, donde los investigadores acompañaron los intentos de los sindicatos locales de influir en el uso de la tecnología en el trabajo, partiendo del principio de que los afectados por un nuevo sistema organizativo deben tener voz en su proceso de diseño.

En los países nórdicos, la legislación y los convenios colectivos reconocían entonces a los trabajadores el derecho a la información y a algún grado de influencia sobre las condiciones tecnológicas de su trabajo. Por lo tanto, se dedicó mucho esfuerzo a diseñar técnicas, herramientas y métodos que les permitieran participar activamente en los procesos de diseño y contribuir a ellos.

Los métodos prácticos, herramientas y técnicas para la cocreación encontraron un campo de ensayo y difusión en este ámbito, y algunos arquitectos, especialmente en los países nórdicos, utilizan sus teorías y experiencias para implementar procedimientos participativos. 
4. Vista aérea y planta de situación. Flexibo, Copenhague, Fællestegnestuen. 1:2000.

5. Emergencia y evolución del sistema Flexibo. Diagrama SCOT.

6. Planta de vivienda tipo 31. Flexibo, Copenhague, Fællestegnestuen. 1:200.
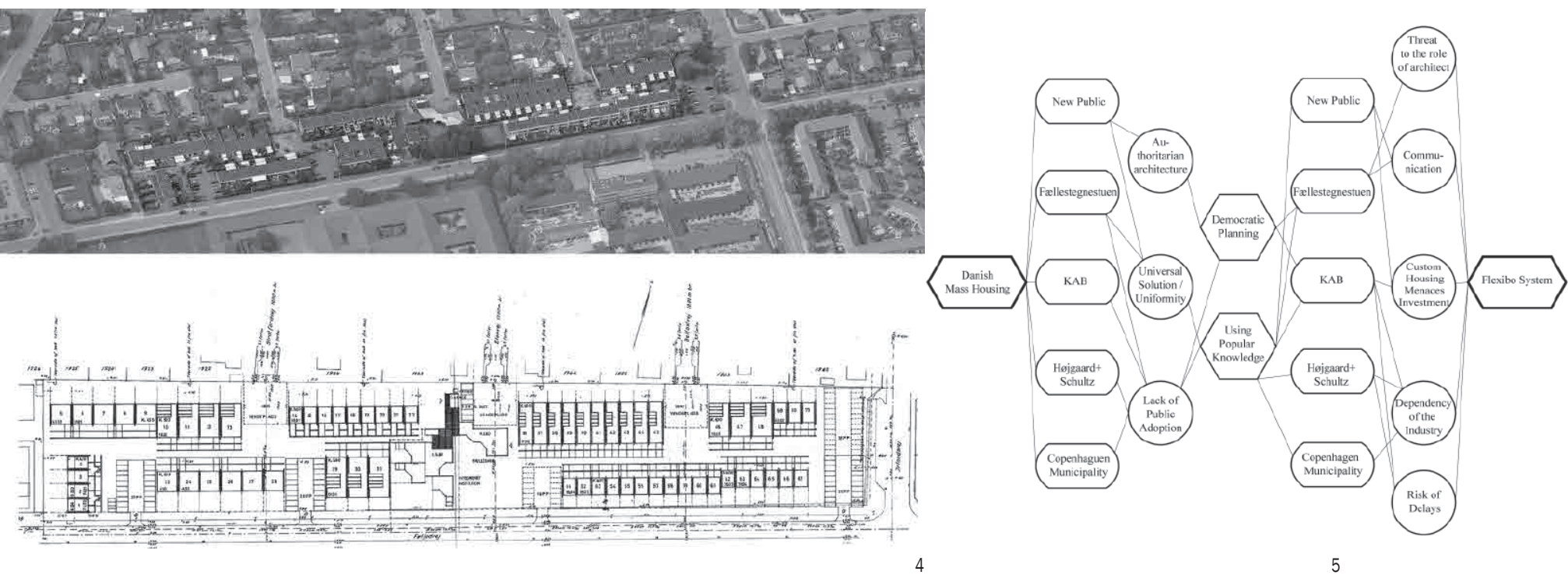

Flexibo

A partir de estas condiciones comparativamente adecuadas, el proyecto de vivienda Flexibo (figura 4) proyectado y construido por la oficina Fællestegnestuen, es propuesto aquí como un caso ejemplar de esa línea de pensamiento arquitectónico que pretende crear dispositivos tecnológicos para la democratización de la vivienda.

Fællestegnestuen ${ }^{25}$ fue fundado por el viejo maestro Viggo Møller-Jensen y otros dos jóvenes arquitectos, a raíz del encargo de la construcción de una ciudad entera para KAB (Asociación de Vivienda Social de Copenhague $)^{26}$, principalmente con el objetivo de experimentar en la industrialización de la vivienda social. Møller-Jensen, activo desde la década de los años 30, fue socio del conocido arquitecto moderno danés Kay
Fisker. La continuidad de la tradición ha sido apuntada como una característica de la modernidad nórdica.

El sistema constructivo de Flexibo, desarrollado por Fællestegnestuen para KAB, fue una de las propuestas que en esa época, en Copenhague, intentaban incrementar la posibilidad de que los inquilinos alterasen sus viviendas $^{27}$. Este proyecto se desarrolla en un contexto de desafecto popular hacia los grandes desarrollos de vivienda producidos en masa en los 60 , percibidos como repetitivos y autoritarios por buena parte del público, y la necesidad económica de mantener la industrialización de la construcción de vivienda, entre otros motivos por el alto coste de la mano de obra local. El sistema ofrece también al arquitecto la oportunidad de proyectar la variabilidad y en cierto grado las decisiones futuras de los inquilinos, reforzando su rol profesional (figura 5).

25. El nombre del estudio tiene ciertas connotaciones democráticas, en danés significa el estudio común (o comunal). 26. PAGH, Jesper. Tyge Arnfred Interview. Arkitektur DK, Copenhague: Arkitektens Forslag, abril 2013, nº 02, pp. 58-63 (p. 60). 27. ØRUM-NIELSEN, Jørn. Dwelling: at home, in community, on earth: the significance of tradition in contemporary housing. Copenhague: The Danish Architectural Press, 1996, p. 204. 
PROYECTO, PROGRESO, ARQUITECTURA

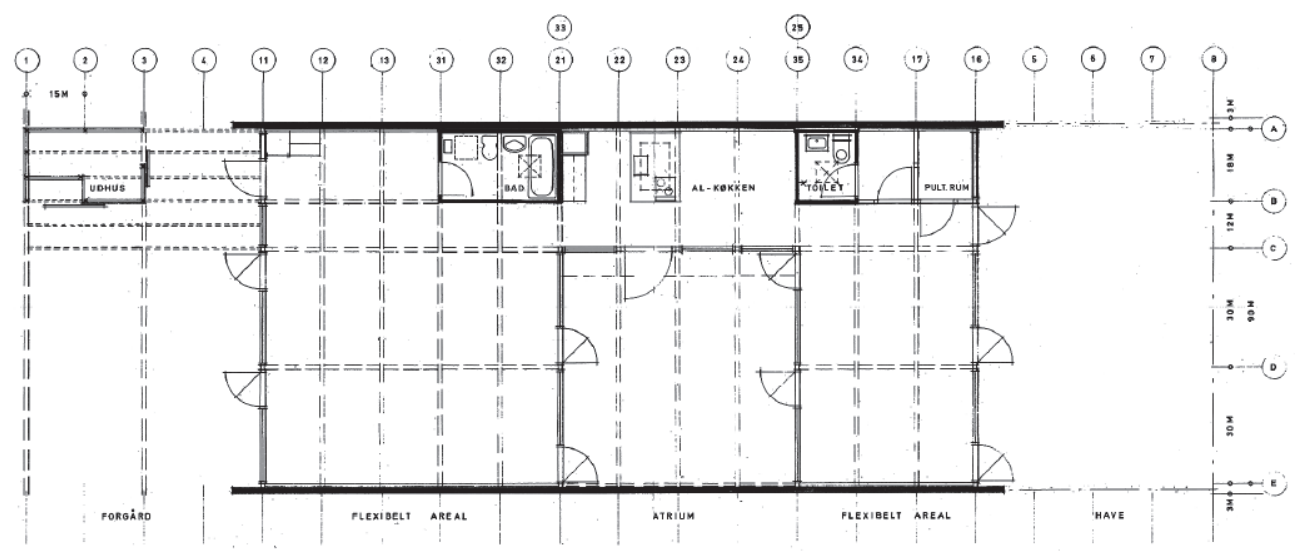

UDHUS = OUTBUILDING

$B A D=B A T H R O O M$

KØKKEN $=$ KITCHEN

PULT. RUM = LUMBER
FORGARD = COURT

FLEXIBELT AREAL = FLEXIBLE AREA

ATRIUM = ATRIUM

6 HAVE = GARDEN

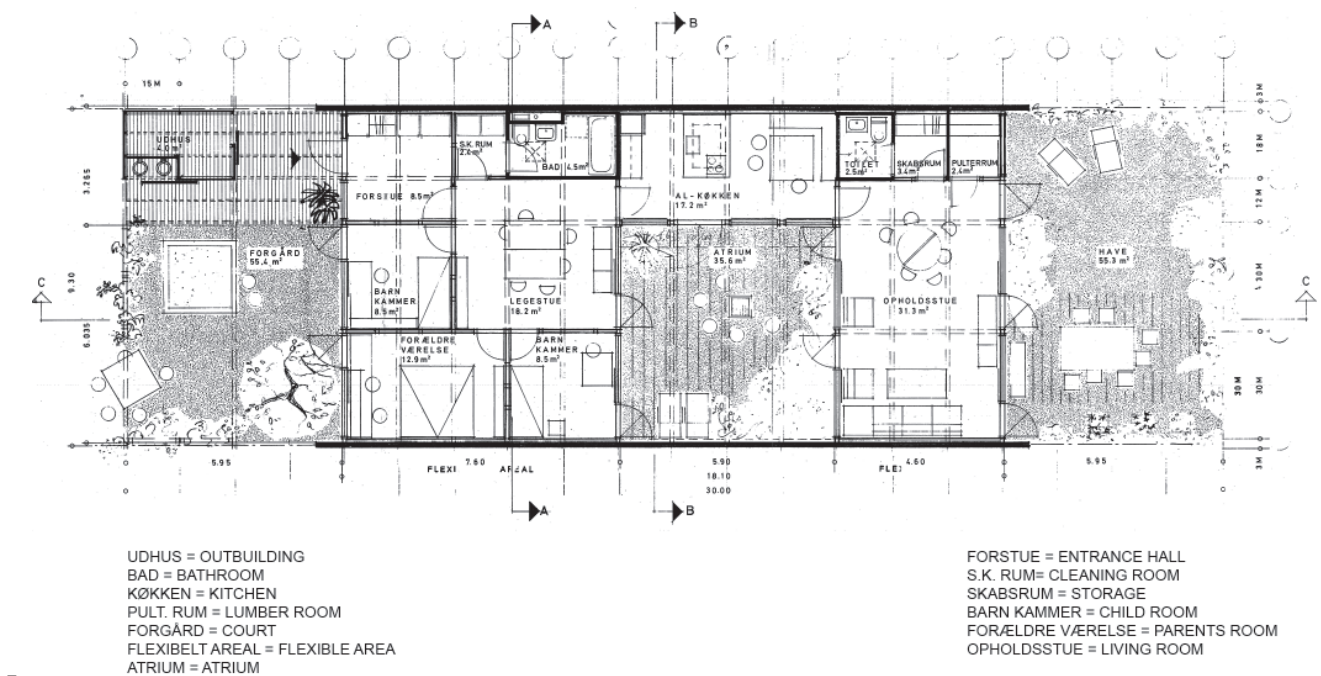

$7 \begin{aligned} & \text { ATRIUM }=\text { ATRIUM } \\ & \text { HAVE }=\text { GARDEN }\end{aligned}$



l BeTONSKEL $_{\text {. }}$

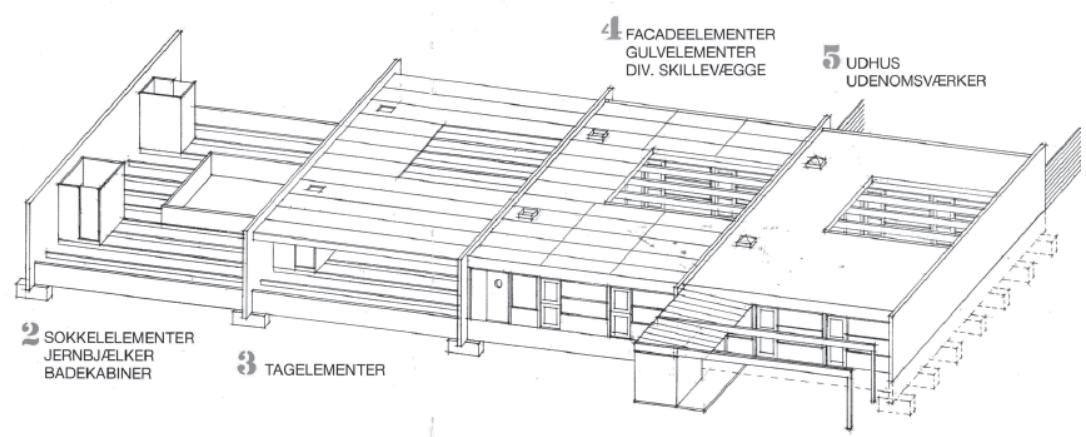

PHASES IN CONSTRUCTION

1. CONCRETE CARCASS

2. SOCLE ELEMENTS, IRON BEAMS, TOILET CABINS

4. FACADE ELEMENTS, FLOOR ELEMENTS, DIVERSE PARTITIONS 


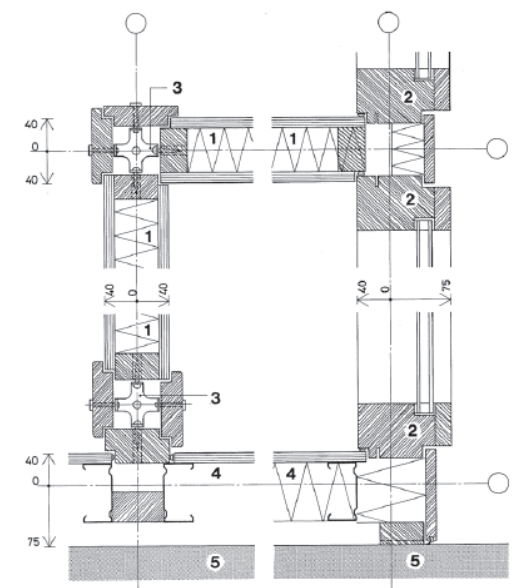

HORIZONTAL SECTION 1:10. JOINT BETWEEN FACADE, CONCRETE SUPPORT AND THE MOVABLE PARTITIONS

1. MOVABLE WALLS

2. FACADE ELEMENTS

3. ASSEMBLY BRACKET WITH CAPPERS SCREWED ON

4. LINER WALL

9 5. CONCRETE WALL

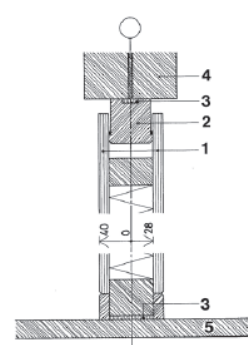

VERTICAL SECTION 1:10. JOINT BETWEEN MOVABLE PARTITIONS, FLOOR AND ROOF

1. $12 \mathrm{MM}$ CHIPBOARD

2. STRIP

4. DOUBLE BEAM OF LAMINATED WOOD OR ADAPTER 5. FLOOR



- NY LEJER

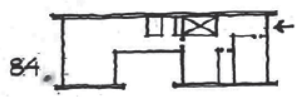

86

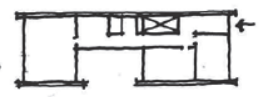

88
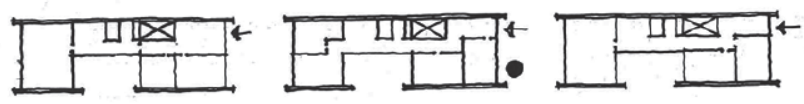

90

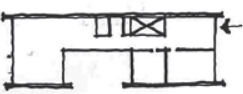

92

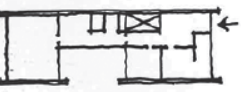

$94 \sqrt{111}$
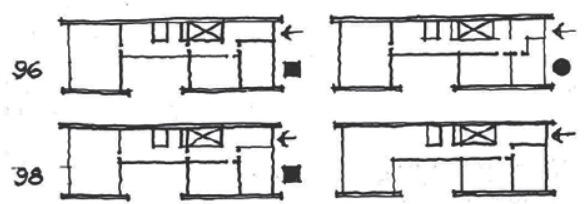

100

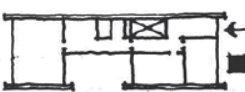

LOR
ANTAL 10 WVORES INDRETNING



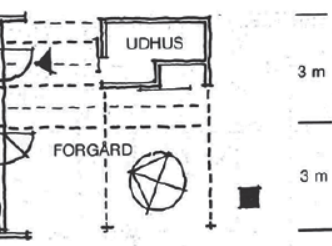

MAX 4 RUM
-
$3 \mathrm{~m}$
-
-
7. Planta de vivienda tipo 31. Distribución recomendada por los arquitectos. Flexibo, Copenhague, Fællestegnestuen. 1:200.

8. Axonometría constructiva. Flexibo, Copenhague, Fællestegnestuen.

9. Detalles constructivos del sistema de particiones móviles. Flexibo, Copenhague, Fællestegnestuen. 1:10

10. Portada del informe de postocupación elaborado por KAB y Fællestegnestuen, junto con evolución de las unidades del tipo de vivienda 31. Flexibo, Copenhague, Fællestegnestuen.

11. Ejemplo de ocupación de vivienda. Annete y Johs Hansen. Flexibo, Copenhague, Fællestegnestuen.

12. La casa común de Flexibo obtuvo un nuevo tejado en los noventa. Sirva simplemente como ejemplo de cómo la concepción del entorno físico, incluso en este contexto, ha permanecido vedada al colectivo por la autoridad de esotéricos criterios profesionales o la expresión estética de ciertos individuos

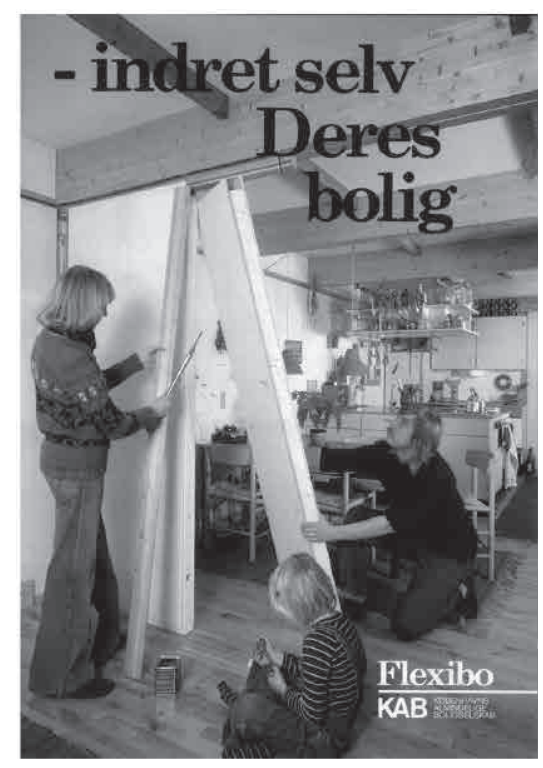

HUSTYPE $=$ HOUSE TYPE ANTAL = NUMBER

RUM = ROOM

NY LEJER = NEW TENANT

VORES INDRETNING = OUR LAYOUT 

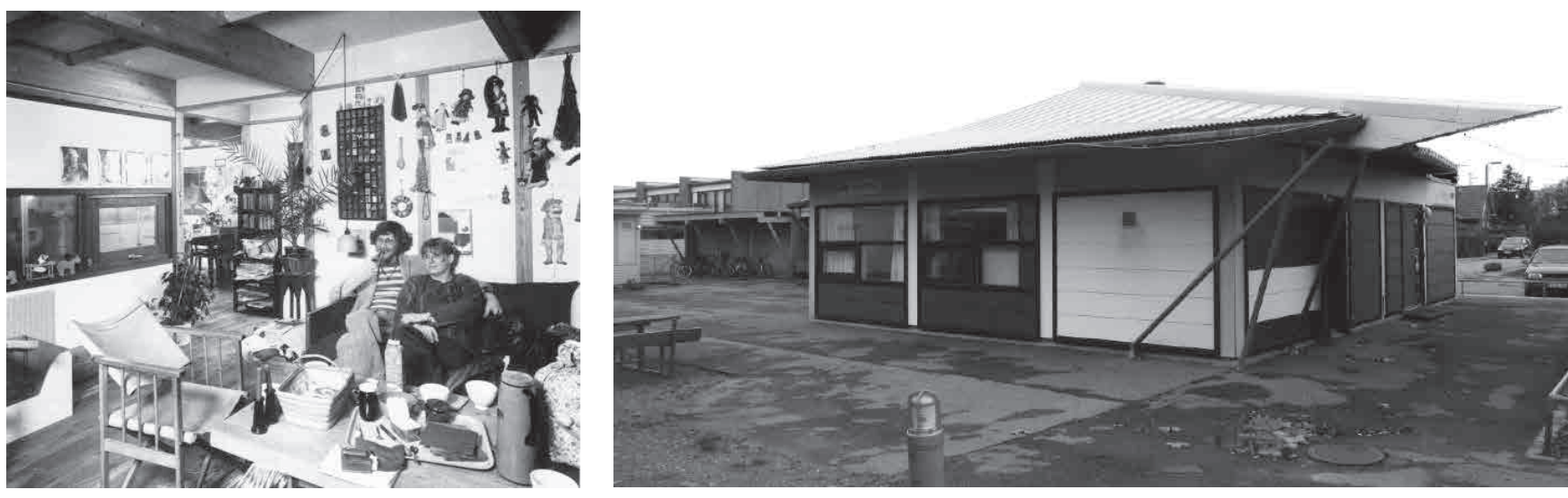

Flexibo fue publicado en medios arquitectónicos en $1971^{28}$ y realiazado por la constructora Højgaard + Schultz en 1976. Forma parte de un esquema de doble escaladonde los arquitectos definen una envolvente y los inquilinos el interior. Se construyeron 68 viviendas en 8 tipos entre paredes paralelas de hormigón prefabricado, además de una casa común y una guardería según los mismos principios constructivos. Los espacios húmedos se ubican junto a los muros. Junto con la envolvente de suelo, cubierta, forjados y fachadas ligeras, son los únicos elementos fijos en unas viviendas totalmente flexibles. (figuras 6 y 7 ).

La innovación del proyecto reside en un sistema de particiones móviles que los propios residentes pueden manejar. Con este sistema, las paredes pueden ser retiradas, recogidas en un almacén o instaladas en poco tiempo sin la ayuda de mano de obra calificada(figuras 8 y 9).

El sistema de paredes móviles, probablemente el principal atractivo de este complejo de viviendas sociales, ha probado su funcionamiento. En 1979, los arquitectos y KAB realizaron una investigación ${ }^{29}$ que demostraba que 40 apartamentos habían sido modificados por los inquilinos, algunos ya varias veces, y sólo 3 inquilinos mantuvieron la disposición recomendada por los arquitectos (figura 10). La gente en realidad reconstruye, reorganiza y mueve las paredes de acuerdo a la evolución de sus gustos o necesidades. Pocos experimentos en vivienda flexible han tenido tanto éxito.

El grado de satisfacción se mostró alto en estos estudios y una larga lista de espera para conseguir un apartamento en la finca lo confirma hoy ${ }^{30}$. Ya en 1980 el sistema fue replicado en otro lugar de la ciudad con un conjunto de 52 unidades. Ambos Flexibos son viviendas sociales, lo que implica su gestión a través de procedimientos de democracia vecinal (figura 11).

Una solución arquitectónica razonablemente eficaz, más allá de su dudoso aislamiento acústico, y un contexto institucional y sociopolítico propicio dieron lugar a un sistema exitoso de vivienda flexible, caso ejemplar de los esquemas de doble escala. No fueron sin embargo suficientes para que el camino a la democratización de la vivienda pasase por estos esquemas, y su impacto sigue siendo modesto. Pese a haber sido canalizadas por procesos bastante democráticos de toma de decisiones, tanto el proceso descrito en Flexibo como especialmente las reformas sucesivas de la edificación han permanecido en gran parte dentro de lo que se habría llamado arquitectura autoritaria en los viejos tiempos (figura 12).

28. Fleksible boliger, Byggesystem for fleksible boliger i æætte, lave, bebyggelser, udviklet for KAB. Arkitekten, Copenhague: Arkitektens Forslag, 1971, vol. 25, pp. 537-45.

29. HOLMBERG, Hartvig, ed. - indret selv Deres bolig. Copenhagen: KAB, 1979.

30. KAB Boligsøgende KAB [accedido 19-03-2018] Disponible en: https://www.kab-bolig.dk/boligsoegende/venteliste-og-ventetid 


\section{DISCUSIÓN: LAS (IM)POSIBILIDADES DE}

\section{LAARQUITECTURA PARTICIPATIVA}

Vistos desde una perspectiva actual, los principales objetivos de la arquitectura participativa, cambiar radicalmente tanto la profesión arquitectónica como el alojamiento humano, no fueron alcanzados. Tanto la sociedad como la cultura arquitectónica dominante los fue dejando en el olvido y no faltaron buenas razones para ello. Por un lado, los proyectos de vivienda participativa tuvieron problemas con:

- Disciplina y profesión arquitectónicas. Los arquitectos inconformistas se organizaron en gran medida como una pequeña vanguardia, ahondando en su propia marginación.

- Estado e industria de la construcción. El contexto económico, con el desmantelamiento del estado de bienestar y el surgimiento de una economía postindustrial no productivista basada en gran parte en la especulación inmobiliaria hizo que estos experimentos de baja rentabilidad económica fueran a menudo inviables. En los países nórdicos, la subida en el precio del petróleo afectó gravemente al muy industrializado sector de la construcción.

- Público general. La condición de las casas como la inversión principal en la vida de la mayoría de los clientes pone barreras importantes para el interés del usuario en la personalización de la vivienda.

- Arquitectos y público comprometidos. Un cierto determinismo arquitectónico y algo de ingenuidad condujeron con frecuencia a grandes expectativas, resultando en grandes decepciones para usuarios y arquitectos, a menudo convertidos en títeres de procesos económicos más amplios que no necesariamente apoyaban.

Por otra parte la cocreación arquitectónica aporta serios problemas internos:

- Técnicos. La comprensión genuina de las necesidades y deseos de una población diversa resultó abrumadoramente difícil, dada la diferencia cultural entre participantes y arquitectos.

- Epistemológicos. Till ha defendido que la plena participación en el diseño arquitectónico, dado el desequilibrio en el conocimiento entre las partes, parece implicar que el arquitecto renuncia a una parte de éste, llevando a una situación de "mínimo común denominador"31, donde "el arquitecto es degradado y el pueblo no accede al poder"32.

- Fronéticos. Como observó De Carlo, el papel del arquitecto en la sociedad dependía de no preocuparse por motivaciones y consecuencias ${ }^{33}$.

Tampoco en los países nórdicos, donde las condiciones fueron más propicias, alcanzó la crítica de los setenta sus objetivos de radical transformación del alojamiento humano y la profesión de arquitecto, más allá de algunos casos marginales. Aunque sutil, su influencia sobre la arquitectura convencional ha sido sin embargo duradera en estos países. Toma la forma de cierta participación institucionalizada en la mayoría de los proyectos urbanos y de vivienda, especialmente en vivienda social, en la permanencia de la democracia vecinal, en la popularización de la covivienda ${ }^{34}$ como un modo de vida convencional y en una cultural general de negociación y compromiso entre los actores del sector de la construcción.

La evolución de Flexibo apunta a una causa de la falta de éxito en la consecución de los objetivos finales de la arquitectura participativa: las iniciativas encaminadas a democratizar la vida cotidiana, por la arquitectura no se acompañaron de proyectos paralelos de democratización de lo más cotidiano de la propia arquitectura: el rol del arquitecto y los procesos creativos permanecieron inalterados.

Al final, aquellos arquitectos, notoriamente en el caso de los sistemas de doble escala, buscaban un imposible: democratizar un aspecto aislado de la vida,

31. TILL, Jeremy. The negotiation of hope. En: BLUNDELL JONES, Peter; PETRESCU, Doina; TILL, Jeremy, eds. Architecture and participation. Abingdon: Spon Press, 2005, pp. 23-41 (p. 25).

32. ROSE, Gillian. Athens and Jerusalem: A tale of two cities. Social and legal studies, Londres: Sage Pub., septiembre 1994, vol. 3, n 3, pp. 333-48 (p. 336). 33. DE CARLO, Giancarlo, op. cit., p. 15.

34. La covivienda es una comunidad de viviendas privadas complementada con extensos espacios comunitarios. Está planeada y manejada por sus residentes, que son habitualmente personas que buscan mayor interacción con sus vecinos. 
la arquitectura, o la vivienda en particular, por medio de unos roles y métodos convencionales. El camino para retomar en el siglo XXI el viejo objetivo de una vivienda democrática que tenga tanto eficacia como agencia social difícilmente podrá ser encontrado en estos esquemas.

Otros proyectos contemporáneos de vivienda en el área nórdica, que tuvieron más difícil encontrar acomodo en el corpus de referencia de la disciplina arquitectónica, se nutrieron también del propicio caldo de cultivo sociopolítico de la época para llevar a cabo experimentos de vivienda bajo el control de sus inquilinos en transición hacia formas antiautoritarias de alojamiento humano donde el sujeto de los procesos constructivos fuera el colectivo.

Experiencias manifiestamente alternativas que desafiaron el papel profesional del arquitecto y los procesos creativos tradicionales, como Tinggården en Herfølge (1978) o Stacken en Gotemburgo (1979), se convirtieron en paradigmáticos de nuevas tipologías de vivienda -bofælleskab y kollektivhus-, a menudo traducidas como covivienda danesa y sueca, respectivamente, mientras que Svanholm (1978) o Christiania (1971) llegaron a ser muy influyentes para las ecoaldeas y los espacios okupados de todo el mundo.

Aun siendo modos de vida minoritarios, su impacto social en la vida urbana general no debe ser menospreciada, sobre todo si se compara con la limitada influencia política que han tenido las tradiciones de arquitectura flexible o participativa. Es más, estas formas de vivienda alternativa respondieron a las nuevas demandas sociales de su tiempo: nuevos modelos familiares, emancipación de la mujer o aislamiento generalizado del individuo, con una imaginación y una agencia social de la que careció de la vivienda convencional.

Estos últimos ejemplos sí abordaron una renovación de la práctica de la profesión arquitectónica constituyéndose en experiencias de infraestructuración ${ }^{35}$ de ocupaciones temporales, contingentes y sociales del espacio.
Recientes movimientos del campo del diseño participativo han evolucionado hacia el "diseño después del diseño", reclamando diseñar para una apropiación y rediseño continuos, resumiendo esta transformación como un paso "desde el diseño de things (cosas) hasta el diseño de Ting (asambleas socio-materiales)" ${ }^{36}$.

Tal vez estos otros experimentos nórdicos, que no pudieron asignarse a sí mismos una tarea menor que "un mundo de su propio diseño" ${ }^{37}$, podrían ayudar a esta generación del siglo XXI a alcanzar el viejo y renovado objetivo de una participación transformadora.

\section{CONCLUSIÓN}

Hay una constelación contemporánea de experiencias, publicaciones, exposiciones y eventos arquitectónicos con enfoques participativos y colectivos, que revelan una corriente subyacente de la sociedad actual con implicaciones para la industria de la vivienda. Una revisión crítica de los pasos en falso de la generación anterior de arquitectos preocupados por la democratización de la disciplina y la profesión en los años sesenta y setenta y su arquitectura de participación revela los muchos problemas internos y externos a los que esta tarea se enfrenta.

Sin embargo, la experiencia demuestra que no fue la reivindicación de la libertad colectiva, sino su evolución en luchas por la autonomía individual y una consiguiente retirada hacia ilusiones de autonomía disciplinar y filosofías de inacción, la razón de su limitado alcance.

La construcción social de la tecnología ayuda a identificar qué problemas afrontó la arquitectura participativa con los principales actores involucrados en el surgimiento y desarrollo de estos dispositivos tecnológicos. Experiencias como Flexibo, donde la constelación de actores y condiciones eran óptimas, apuntan a un problema crucial: los esfuerzos por la democratización de la vida cotidiana por la arquitectura no fueron acompañados por

35. Infraestructuración es un término de la teoría del diseño participativo asociado al trabajo teórico de Stan Allen. En este contexto, infraestructuración debe entenderse como un proceso prolongado de colaboración técnica en la creación de un sistema, más allá de su limitación a los procesos de la fase de diseño, como en los casos de doble escala. BJÖGVINSSON, Erling; EHN, Pelle; HILLGREN, Per-Anders. Design Things and Design Thinking: Contemporary Participatory Design Challenges. En: Design Issues, Cambridge (MA): MIT Press, verano 2012, vol. 28, nº 3, pp. 101-16.

36. Ibíd., p. 102. Alude a los escritos de Bruno Latour sobre la etimología de la palabra inglesa thing como asamblea en las antiguas sociedades germánicas. 37. DEBORD, Guy. The society of the spectacle. London: Boureau of Public Secrets, 2014, p. 63. 


\section{N18_ARQUITECTURAS AL MARGEN}

56

esfuerzos paralelos hacia la democratización de lo más cotidiano de la arquitectura: el papel del arquitecto y los procesos creativos.

En vez de reiterar las tácticas fallidas del pasado, una desnaturalización de lo más cotidiano de la profesión arquitectónica a través de una infraestructuración de las prácticas de vivienda por parte de arquitectos e inquilinos podría retomar los viejos y populares objetivos de integrar procesos de aprendizaje mutuo y fomentar las prácticas democráticas en la vida cotidiana.

\section{Bibliografía citada:}

AIBAR, Eduardo; BIJKER, Wiebe E. Constructing the city: The Cerdá Plan for the extension of Barcelona. Science, Technology and Human Values. Sage Pub., enero 1997, vol. 22, nº 1, pp. 3-30. ISSN 0162-2439. D0l: https://doi.org/10.1177/016224399702200101

ARNSTEIN, Sherry R. A ladder of citizen participation. Journal of the American Planning Association, American Planning Assoc., vol. 35, n 4 , pp. 216-24. ISSN 0194-4363. D0l: http://dx.doi.org/10.1080/01944366908977225

BLUNDELL JONES, Peter. Sixty-eight and after. En: BLUNDELL JONES, Peter; PETRESCU, Doina; TILL, Jeremy, eds. Architecture and participation. Abingdon: Spon Press, 2005, pp. 127-40.

BJÖGVINSSON, Erling; EHN, Pelle; HILLGREN, Per-Anders. Design Things and Design Thinking: Contemporary Participatory Design Challenges. Design Issues, Cambridge (MA): MIT Press, verano 2012, vol. 28, n³ 3, pp. 101-16. ISSN 0747-9360. D0l: https://doi.org/10.1162/DESI_a_00165

BOOKCHIN, Murray. Social anarchism or lifestyle anarchism. An unbridgeable chasm. Edinburgh - San Francisco (CA): AK Press, 1995.

CRAWFORD, Margaret. Can architects be socially responsible? En: GHIRARDO, Diane, ed. Out of site: a social criticism of architecture. Seattle: Bay Pres, 1991, pp. 27-45. ISBN 9780941920193.

DEBORD, Guy. The society of the spectacle. London: Boureau of Public Secrets, 2014.

DE CARLO, Giancarlo. Architecture's public. En: BLUNDELL JONES, Peter; PETRESCU, Doina; TILL, Jeremy, eds.. Architecture and participation. Abingdon: Spon Press, 2005, pp. 3-22. 
PROYECTO, PROGRESO, ARQUITECTURA

57

ELLIN, Nan. Participatory Architecture of the Parisian periphery: Lucien Kroll's Vignes Blanches. Journal of Architectural Education, Routledge, primavera 2000, vol. 53, n 3, pp. 178-83. ISSN 1531-314X. D0I: https://doi.org/10.1162/104648800564572

FLYVBJERG, Bent. Phronetic Planning Research: Theoretical and Methodological Reflections. Planning Theory and Practice, Routledge, 2004, vol. 5, n 3, pp. 283-396. ISSN 14649357. DOl: https://doi.org/10.1080/1464935042000250195

HABRAKEN, Nicholas J. Supports: an alternative to mass housing. 2. ed. London: Urban International Press, 1999.

HARRIS, Richard. Slipping Through the Cracks: The Origins of Aided Self-help Housing, 1918-53. En: Housing Studies, Londres: Carfax Publishing Ltd. 1999, vol. 14, nº 3, pp. 281-309. ISSN 0267-3037. D0I: 10.1080/02673039982803

HARVEY, David. The New Urbanism and the communitarian trap. En: Harvard Design Magazine, [en línea]. Cambridge (MA): Harvard University Graduate School of Design, 1997. n 1[consulta: 19-03-2018]. ISSN 1093-4421. Disponible en: http://www.harvarddesignmagazine.org/issues/1/ the-new-urbanism-and-the-communitarian-trap

HOLMBERG, Hartvig, ed. - indret selv Deres bolig [Distribuya su vivienda usted mismo]. Copenhagen: KAB, 1979.

JENSEN, Lotte; KIRKEGAARD, Ole; PEDERSEN, Dan Ove. Beboerdemokrati og forvaltning i den almene boligsektor. SBi-rapport 332 [Democracia vecinal y administración en el sector de la vivienda social. Informe 132 del Instituto Nacional de la Construcción]. Copenhagen: SBi, 1999.

KAMINER, Tahl. Architecture, crisis and resuscitation. New York: Routledge, 2011.

KIRKEBY, Inge Mette Knowledge in the making. Architectural Research Quarterly, Cardiff: Welsh School of Architecture, diciembre 2009, vol. 13, ${ }^{\circ}$ 3/4, pp. 307-13. ISSN 1359-1355. D0l https://doi.org/10.1017/S1359135510000151

MANNICHE, Peter, Living democracy in Denmark: independent farmers, farmer's cooperation, the folk high schools, cooperation in towns, social and cultural activities, social legislation, a Danish village. 2. ed. Copenhagen: G.E.C. Gad Pub., 1970.

PAGH, Jesper. Tyge Arnfred Interview. Arkitektur DK, Copenhague: Arkitektens Forslag, abril 2013, nº 02, pp. 58-63. ISSN 0004-2013.

QUERRAIN, Anne. How inhabitants can become collective developers: France 1968-2000. En: BLUNDELL JONES, Peter; PETRESCU, Doina; TILL, Jeremy, eds. Architecture and participation. Abingdon: Spon Press, 2005, pp. 105-115.

ROSE, Gillian. Athens and Jerusalem: A tale of two cities. Social and legal studies, Londres: Sage Pub., septiembre 1994, vol. 3, n 3, pp. 333-48. ISSN 09646639. D0I: http://journals.sagepub.com/doi/abs/10.1177/096466399400300302

SCHNEIDER, Tatjiana; TILL, Jeremy. Flexible housing. Oxford: Architectural Press, 2007.

TILL, Jeremy. The negotiation of hope. En: BLUNDELL JONES, Peter; PETRESCU, Doina; TILL, Jeremy, eds. Architecture and participation. Abingdon: Spon Press, 2005, pp. 23-42.

TZONNIS, Alexander. Community in the mind. A model for personal and collaborative design. CAADRIA 2000 Proceedings of the Fifth Conference on Computer Aided Architectural Design Research in Asia, Singapore, numerous 18-19, May 2000, pp 1-14.

WEIL, Simone. Nota sobre la supresión de los partidos políticos. En: WEIL, Simone; VALLS, Sylvia María (ed.). Profesión de fe. Antología y crítica alrededor de su obra. Versión corregida y aumentada. México: Pleroma, 2007.

ØRUM-NIELSEN, Jørn. Dwelling: at home, in community, on earth: the significance of tradition in contemporary housing. Copenhague: The Danish Architectural Press, 1996.

Fleksible boliger, Byggesystem for fleksible boliger i tætte, lave, bebyggelser, udviklet for KAB [Viviendas flexibles, sistema constructivo para viviendas flexibles en edificios de vivienda de alta densidad y baja altura, desarrollado para KAB]. Arkitekten, Copenhague: Arkitektens Forslag, 1971, vol. 25, pp. 537-545. ISSN 004-198X.

KAB Boligsøgende [buscador de vivienda social], KAB (Asociación de vivienda social de Copenhague) [accedido 19-03-2018] Disponible en: https:// www.kab-bolig.dk/boligsoegende/venteliste-og-ventetid

Rodrigo Rieiro Díaz (Madrid, 1982) studied architecture in Madrid, Barcelona, and Budapest. He practised in Madrid, Istanbul, and Copenhagen, and researches a Ph.D. at Escuela Politécnica de Arquitectura de Madrid. He recently completed a research stay at Statens Byggeforskningsintitute in Copenhagen.

Kim Haugbølle (Stege, 1965) conducts advisory services to the government, undertakes teaching, and develops research-based knowledge to improve building environment. He has authored or co-authored more than 160 publications on the role of construction client focusing on innovation, procurement, building performance, life cycle economics, and sustainable design. 
(IM)POSIBILIDADES DE LA VIVIENDA PARTICIPATIVA: RETORNANDO AL SISTEMA FLEXIBO (IM)POSSIBILITIES OF PARTICIPATORY HOUSING: REVISITING THE FLEXIBO SYSTEM

Rodrigo Rieiro Díaz, Kim Haugbølle

\section{p.43 INTRODUCTION}

Housing, as well as architecture in general, has often been presented as a post-political issue, i.e. beyond the traditional mechanisms of organisation of the public sphere. For many, it would be either technically or economically determined, notably during the previous decades of withdrawal of the political dimension of architecture.

Others have addressed housing as a political responsibility. The role of architects in the betterment of society by a process of redirecting our technological systems in ways inspired by democratic principles has been a recurrent matter of concern of the architectural discipline and profession. This is most apparent by that tradition of the Modern Movement, which rises against the inflexibility and authoritarism of total design and Gesamtkunstwerk ${ }^{1}$ and finds expression in the modern conception of space offering an equal and neutral stage for life to happen.

There has been a minor but recurring stream of architects and a part of the public in many countries willing to incorporate dwellers and professionals together in the conception of housing. It came to the forefront of the architectural debate hand in hand with citizen participation during the 1960s and 1970s. However, the success of this endeavour has been rather limited so far and the democratisation of the processes of human accommodation remains a pending task.

This is the backdrop set for a new generation of architects, who would be renewing and rethinking those controversies, supported by a growing interest in alternative architectures by part of the public in the post-2008 financial crisis, post-housing bubble context.

This paper revisits the tradition of participatory architecture from the 1960 s and 1970 s, focusing on the particularly beneficial socio-historical conditions it found in the Nordic countries. This paper argues that one successful exemplar of this trend, Flexibo in Copenhagen, epitomises the crucial flaw of this development: ironically, it tried to implement democratic housing by authoritarian means and processes.

Other resourceful practices in this field in the Nordic countries, hitherto little known in Spanish or English-speaking academic circles, point to a twenty-first century architecture of participation aimed to lift the average quality of everyday

p.44 building practice for the benefit of all. Could these marginal experiments of the Nordic countries help this twenty-first century generation to achieve the revived old goals of democratic ${ }^{2}$ housing?

\section{METHODOLOGICAL CONSIDERATIONS}

Theory

Participatory architecture, as it has developed historically, implies a characterisation of architectural knowledge as phronetic ${ }^{3}$, i.e. as a practical wisdom standing on the shoulders of scientific and technical knowledge directed towards a good life.

In turn, that entails an understanding of architectural works as techno-political constructs. Social constructivism of technology offers a useful framework for studying the emergence and evolution of these constructs. Indeed, some of its main advocates have demonstrated the suitability of this methodology for the study of the politics of architecture. ${ }^{4}$

Critiques have been voiced too on the limitations of its inherent reductionism. This paper is also concerned with a wider and deeper understanding of the dynamics evident in the architectural works. Therefore, a loose nondeterministic materialist position underlay the text, in that diverse tradition in which superstructure and base mutually determine and interrelate.

The Nordic tradition of participatory design offers theoretical underpinnings for the congruity of this commitment, phronetic knowledge and co-creation. ${ }^{5}$ Furthermore, an evaluative stance of what the studied case means in terms of the qualities of everyday living, the broader distribution of power in society and the collective freedom has been considered essential coherent with the object of study.

\section{Field of study}

Firstly, the scope of the study has been limited to housing. Housing has been the main subject of architecture, at least as the profession are established from the Modern Movement. It was also the main matter of concern for the architects and the public committed to participation in architecture in the 1960s and 1970s. In the same period, there is high prevalence of housing not designed by architects, and for very different reasons. Last, but not least, it posits technically complex problems from a very small scale, pleading for the help of a professional, while it provides an issue on which non-professionals often have a broad practical knowledge.

'Participatory housing' is still a rather vague concept. The guideline to narrow the field of study has been restricting it to cases where the subject of the constructive action were non-exclusive collectives of professionals and non-professionals together. Probably, there is always some level of participation in decision-making in all human endeavours of such complexity like housing. Therefore, it makes little sense to speak in binary terms (of participatory or non-participatory processes). The focus has been directed towards experience ranking high in Arnstein's canonical 'ladder
participation', 6 leaving aside cases where participation is not accompanied by high levels of decision-making 
Methods

This paper is primarily the result of a critical review of the available literature on the tradition of participatory housing from the 1960 s to the present ${ }^{7}$ There is a proud history of citizen involvement in the Nordic countries, and it is therefore a tradition encouraging participation in many fields, including those related to housing.

Hence, this study zooms in on a case study of a prominent Danish example of participatory architecture in housing development. The housing project Flexibo is an exemplary case of this participatory housing tradition, but at the same time it also points to some of the flaws, drawbacks and dilemmas of the participatory housing tradition. Data for this study were obtained by visits to the actual site, local architectural publications from the 1970s and 1980s, and technical documentation. Technical documentation was accessed via the physical archive of the Centre for Buildings (Center for Bygninger) of the municipality of Copenhagen.

\section{PARTICIPATORY HOUSING: EMERGENCE AND PRINCIPLES}

The emergence of and principles of participatory housing is part of a more general history of democratisation in architecture, in everyday life, at home, at work, and in the neighborhood. The next section gives a brief overview of the development within the three first areas that together form the backdrop for understanding the emergence of participatory housing.

Figure 1 provides an overview of the most important relevant social groups, the main problems and solutions, and resulting artefacts in relation to the 1970s participatory housing. One relevant social group consisting of a select part of the public and small group of architects in favour of participatory housing identified one set of problems like imbalance of power. The other and much larger relevant social group of the general public and mainstream architects in unison with the state and the construction industry faced other problems, like unfeasibility in the context of a shrinking welfare state. The realised solutions led to new technological artefacts in which an exclusive group of the public prevailed, participation was very low, or architects and users shared the different phases of housing planning (figure 1).

\section{Democracy in architecture}

At the turn of the 1960s and the 1970s, it became increasingly popular in the architectural culture of first-world nations to question housing for the working-class as planned 'for the worker, not by the worker'. As stated in the seminal manifesto Architecture's Public ${ }^{8}$ by Giancarlo de Carlo, the project of a 'liberating and democratic' architecture of participation ${ }^{9}$ was not only about giving political legitimacy to planning, but also about radically transforming the discipline and the profession. This involved going beyond the how, where architects have typically found their sustenance, to re-address the why - and the what - to do in architecture. To put it in Aristotelian terms, it meant to go beyond the techne (technical know-how) and the episteme (scientific knowledge) types of knowledge to bring back phronesis (practical wisdom, relying on the other two).

Around the 1970s, some housing estates were planned to varying extents by and with their inhabitants and architects together. The Nuovo Villaggio Matteotti in Terni by Giancarlo de Carlo, the Byker Wall by Ralph Erskine, the Medical Faculty Housing in Louvain by Lucien Kroll, the Ökohaus in Berlin by Frei Paul Otto and the self-built houses in Lewisham by Walter Segal are probably the most published developments when it comes to participatory housing in the architectural media.

Margaret Crawford argued that the radical architects' claims were in fact an incomplete negation, simply reversing the already fictional roles of the all-powerful architect and the ideal client - the masses - while accepting the ideological assumptions on which they rested. Facing their inefficiency to effect social changes, they increasingly focused on mere opposition to the aesthetics of modernism. Therefore, they replaced modernism's welfare state with a marketplace in which their ideal client did not have the means to purchase architectural services ${ }^{10}$.

Lack of social agency ${ }^{11}$ led to an aestheticisation of the political. Tahl Kaminer unfolded how the dead-ends of the architecture of the 1970s led it to a withdrawal in the autonomy of paper architecture and then to the present scenario of star architects $^{12}$

Politics in housing evolved towards spectacle too. On the one hand, some saw participation as a means to build consensus, giving a feeling of having influenced the decisions, and having a positive factor in the acceptance of decision outcomes. This pseudo-participation ended by making it even more difficult to achieve authentic participation in some contexts ${ }^{13}$.

The political dimension of participatory housing design was also exhausted by invoking the myth of the uniform community. In Community Architecture ${ }^{14}$, both architect and citizens' authorities were dissolved in the name of the myth of the 'purified community', 'defined by groups of a narrow social definition, at worst driven by NIMBY selfinterest, and therefore exclusive',

The approach of the previous generations of architects committed to participation led them to social inefficiency The 'thorough reformulation of both theory and practice in order to avoid repeating the well-intended but mistaken strategies used by modernist reformers and sixties radicals, 16 remains a task as pending as it was when Crawford 
formulated it twenty-five years ago. The spectacular landscape of the contemporary urban developments, where iconic buildings complement the superficial variation in the facades of otherwise repetitive housing units, well represents the liberal democratic ethos those non-conformists had opposed.

\section{Democracy in the everyday}

The relationship between architecture and everyday life ${ }^{17}$ shapes whether participatory housing can be a democratising endeavour at all. It was precisely through a personal commitment with the everyday that a part of that generation aimed to attain practical utopias. A de-naturalised everyday would demand judgement. It is in this way that each and everyone has a relationship with the everyday that, following the feminist maxim, the personal becomes the political. Accordingly, the type of practical knowledge may mean a focus on everyday life, everyday people and the ordinary. To retreat from this has been understood by some architects, notably in the 1970s, as an abrogation of professional responsibilities.

The growing demand for freedom, individualism and creativity by the post-Fordist society stressed the acceptance of the artistic critique of society and political impotence led to the retreat from the social critique. In the everyday architecture done by non-professionals, some architects identified spaces in which difference and 'the other' might flourish.

At the time, the appeal of the 'non-pedigreed' building had been underlined by the popularity of Rudofsky's exhibition and book, Architecture without architects, and Blundell Jones notes how in Boudon's popular book about Le Corbusier's Pessac ${ }^{18}$, 'owner's alterations to the Pessac housing were taken seriously, and that the author dared to compare the people's expression with that of the master'. The sense of spontaneity, invention and creativity evinced in such 'bricolaged' work was aesthetically appealing as an antidote to the unpopular rationalist, industrialised work of establishment architects.

However, there is a fundamental difference between the freedom of collective self-organisation and the individual freedom from society's edicts and restrictions. Bookchin has accounted for the big chasm existing between the personal commitment to the individual autonomy and the collectivistic commitment to social freedom. He traced it to the opposition between the word liberty from the Roman Imperial 'libertas', 'wherein the untrammelled ego is "free" to own his personal property', and the Germanic-rooted word freedom 'where the selfhood does not stand opposed to or apart from the collective but is significantly formed by his or her own social existence, ${ }^{19}$. It was not the demand

p.48 for collective freedom per se, but its evolution into struggles for individual autonomy that explains the withdrawal into aesthetic criticism.

\section{Democracy at home}

Some architects have seen the solution to the many troubles of participatory architecture in the creation of a sort of double-scale architecture. It keeps authoritarian approaches in the conception and construction phases, when the architect's role is traditionally prevailing, while defining the conditions to high levels of decision-making for residents during the rest of the life cycle of the building - when architects' presence was already typically minor.

The idea was clearly articulated in Habraken's 1961 work: Supports: an alternative to mass housing ${ }^{20}$. This work laid out a system in which the support structure, both technical device and social frame, is set apart from infill or interior fit-out in housing construction and design, to facilitate his ideas of user participation. This double-scale architecture also accords with an isotropic conception of space, with many precedents in Modern Movement history ${ }^{21}$.

This path can later be followed to the raw finishes of the houses of Lacaton and Vassal or to the awarded Quinta Monroy Housing by Elemental and Alejandro Aravena. In this project of social housing, architects design and professionals build a basic shelter, all supported by the state, which is to be completed by the inhabitants on their own. Being the participatory endeavour aimed mainly to transform welfare state-supported housing, it unsurprisingly turned its eyes to the other main institution-supported housing system emerging in the world. It is impossible to ignore the affinity between the support+infill system and the precedent aided self-help approaches ${ }^{22}$.

\section{REVISITING THE EXEMPLARY CASE OF THE FLEXIBO SYSTEM}

Before revisiting the Danish Flexibo, it is important to shed light on two areas with significant peculiarities in the Nordic countries, tenant's democracy and workplace democracy. It helps to understand the emergence of the Flexibo system in Denmark as an exemplary case of participatory housing.

\section{Tenant's democracy}

Some particularities of the Danish housing system led highly democratic practices of housing by non-exclusive groups of architects and tenants in unison to be realised. First and foremost, the line of work by philanthropic societies and mutual aid working-class organisations that sprung up in the nineteenth century was never completely co-opted by the state. It belongs to a wider context of a culture of living together, sharing, and working horizontally that characterises Denmark at least since the influence of the Danish Cooperative Movement (in Danish: andelsbevægelsen) and Grundtvig's pedagogy during the $19^{\text {th }}$ century $^{23}$ (figure 2).

The Danish cooperative movement, which follows parallel developments in the other Nordic countries, was primarily a peasant and religious movement that transformed the Danish countryside. Its urban version, linked to the rise of the international working class movement, reached a huge impact on the housing sector by the housing 
cooperatives (andelsbolig), which together with the also self-managed social housing (almen bolig), today make up 26 per cent of Danish housing - peaking at 37 per cent in Copenhagen (figure 3).

Some features of Danish society helped these experimental utopias of the late 1970 s to be realised. These include institutional support in the context of a universal welfare system, a comparatively egalitarian society, high levels of economic independence and self-management in housing, and the then emerging Danish specific concept of tenants' democracy - which today by law rules all social housing in the country.

'Beboerdemokrati' (tenants' democracy) is also a legacy of the Danish $19^{\text {th }}$ century housing cooperative tradition, characterised by high levels of tenants' control at an estate scale. With the Housing Provision Act of 1970 tenants were allowed to run their estates themselves through democratically elected tenant boards. In 1984, the Law on Tenants' Democracy confirmed it as the main feature of the Danish social housing system. 20,000 tenants of approx. 1 million tenants in total hold an elected post, 33 per cent of all tenants participate in the annual meetings of the housing estates, and tenants perform 10 per cent of maintenance work during common working days ${ }^{24}$.

\section{Workplace democracy}

The other particularities is the principle of involving users in the processes of design, which has been more clearly articulated outside architectural discipline by the field of participatory design that emerged among the various social and political movements of the 1960s and 1970s, when many people demanded an increased say in the decision-making of different aspects of their life.

Participatory design has its roots in the movements towards democratisation of work places in the Nordic countries, particularly Sweden, in which researchers supported the attempts of local trade unions to influence the use of technology at work, from the standpoint that those affected by a new approach should have a saying in the design process.

In the Nordic countries, legislation and workplace agreements allowed workers the right of information and some degree of co-determination over the technological conditions of their work. Therefore, much effort was spent on design techniques, tools, and methods that would enable workers to actively participate in and contribute to the design processes.

Practical methods, tools, and techniques for co-creation found a field for testing and spreading in this field, and some architects, especially in the Nordic countries, make use of both of these theories and practices to implement participatory procedures.

The housing project Flexibo

Out of these comparatively favourable conditions, the housing project Flexibo (figure 4) designed and built by the office Fællestegnestue is an exemplar of that train of architectural thought that aims to create technological devices for the democratisation of the everyday by authoritarian structures and processes.

Fællestegnestuen ${ }^{25}$ was founded in the wake of the commission of an entire town for KAB (Copenhagen's Social Housing Association) ${ }^{26}$ by the old master Viggo Møller-Jensen, active from the 1930 s, partner of the famous Danish architect Kay Fisker, and two younger architects, mainly to experiment with industrialisation in social housing. The continuity of tradition has been pointed as a characteristic of Danish modernity.

Flexibo's constructive system was developed by Fællestegnestuen for KAB. It is one of the varied proposals of that time in Copenhagen trying to increase tenant's capacity to modify their own dwelling environments ${ }^{27}$. They emerged from the confluence of a popular disaffection for the mass-produced housing developments of the 1960s, perceived as repetitive and authoritarian by a good part of the public, and the economic need to retain the industrialisation of housing construction, one reason being the high labour cost in Denmark. The system offers the architect the opportunity to project variability and, to a certain degree, tenant's choices into the future, strengthening his professional role (figure 5).

Flexibo was published in the architectural media in $1971^{28}$, and was built by the construction company Højgaard+Schultz in 1976. It belongs to a double scale approach with architects defining the supporting structure and tenants the infill. 68 houses distributed on 8 different types were constructed with parallel dividing walls of prefabricated concrete. Flooring, roof, deck elements and the light facades, as well as wet rooms and kitchens placed along the partitions, are the only fixed elements in the otherwise flexible house (figure 6 and 7).

The innovation in the building is a system for moveable partitions, which the residents themselves can manage. With this system, walls can be moved, stored in a storeroom and set up in a short time without the assistance of skilled labour. Outer structures are also designed to be partially self-constructed by the residents (figure 8 and 9).

The flexible wall system is probably the main attraction of this social housing complex and it has proved to work. In 1979, the architects and KAB conducted a survey showing that 40 apartments had been modified by tenants - some of them several times already - and only 3 tenants kept the architects' recommended arrangement (figure 10) ${ }^{29}$. People actually rebuild, re-arrange and move walls. Few experiments in flexible housing have proved so successful.

The degree of satisfaction proved high in earlier studies, and a long waiting list to get an apartment on the estate confirms it today ${ }^{30}$. As early as in 1980, the system was replicated with 52 houses in another suburb of the metropolitan area of Copenhagen. Both Flexibos are social housing estates, implying enforcement of tenants' democracy policies (figure 11).

A rather efficient architectural solution, beyond the dubious acoustic insulation, and a favourable institutional and socio-political context let a successful flexible housing system emerge as an exemplar of the double scale schemas. 
However, that was not enough to lead the democratisation of the housing endeavour towards these approaches, and its impact remains limited. Despite being channelled by the highly democratic decision-making processes, even the successive reforms of the common spaces of Flexibo have remained largely under the realm of what would have been called authoritarian architecture in the old days (figure 12).

\section{p.54 DISCUSSION: THE (IM)POSSIBILITIES OF PARTICIPATORY ARCHITECTURE}

The main goals of participatory architecture regarding the radical transformation of both architectural profession and human accommodation were not achieved. Both mainstream architectural culture and the society in general let them fall into oblivion. It did not happen without good reasons. On the one hand, participatory housing projects faced problems with:

- Architectural profession. The non-conformist architects were largely organised as a small vanguard, resulting in their marginalisation.

State and the construction industry. Economic context, with the dismantling of the welfare state and the rise of a non-productivist post-industrial economy based largely on real estate speculation often made these low-return experiments infeasible. Specific to the Nordic countries, the rise in oil prices strongly affected the energy-intensive industrialised housing sector.

General public. The condition of houses as the main investment in most clients' lifespans put important barriers for the user' interest on housing customisation.

Committed architects and public. Inherited architectural determinism and a certain naivety frequently led to high expectations resulting in disappointment for users and architects, who often became puppets in socio-economic market processes that they may not support.

Yet on the other hand, participatory design in architecture entails some problems in itself:

- Technical problem. Genuine understanding of the varied needs and demands of users proved daunting, given the difference between the participants and the architects' backgrounds.

Epistemological problem. Till argued that full participation in architectural design, given the imbalance in knowledge of the parties, seems to imply that the architect relinquishes part of it, leading to a 'lowest common denominator' situation ${ }^{31}$, on which ' $(t)$ he architect is demoted; the people do not accede power'32.

Phronetical problem. As De Carlo observed, the role of the architect in society depended on not worrying about motivation and consequences ${ }^{33}$

Not even in the Nordic countries, where conditions were comparatively appropriate, did the criticism of the 1970s reach its goals of radical transformation of the human accommodation and architectural profession, beyond some marginal cases. Yet subtle, its influence on mainstream architecture has nevertheless been long-lasting in these countries. It takes the form of some institutionalised participation in most urban and housing projects, notably in social housing, in the permanence of tenants' democracy, the popularisation of co-housing ${ }^{34}$ as a conventional way of life, and in an overarching culture of compromise and negotiation among the different actors of the construction sector.

The evolution of Flexibo points to a more principal cause of the lack of success in achieving the final goals of participatory architecture: the efforts towards the democratisation of everyday life by architecture came unaccompanied by parallel efforts towards democratisation of the 'everydayness' of architecture: architect's role and creative processes remain unaltered.

In the end, those architects, blatantly in the double-scale cases, were seeking the impossible: to democratise an isolated aspect of life, architecture, or housing in particular, by unchallenged means and roles. Hence, reworking the old goal of democratic housing with both social agency and efficiency can hardly be found in these tactics in the twenty-first century.

Contemporary with Flexibo, other housing projects in the Nordic area, which had a harder time finding a place in the reference corpus of the architectural discipline, became experiments on mutual aid devices to transform housing under tenants' control in transition towards an anti-authoritarian way of human accommodation, where the subject of the construction processes was the collective.

Alternative cases like Tinggården in Herfølge (1978) or Stäcken in Gothenburg (1979) challenged the architect's professional role and the traditional creative processes, and they became paradigms of new housing typologies - bofælleskab and kollektivhus, often translated respectively as Danish and Swedish co-housing. Other examples like Svanholm (1978) or Christiania (1971) became highly influential for eco-villages and empty buildings and land occupied by squatters around the world.

Although these examples represent minority ways of life, their social impact in the mainstream urban life should not to disregarded, especially compared with the limited political influence that flexible and participatory architecture traditions had worldwide. Furthermore, these alternative housing models responded to new societal demands of their time - new family models, women's emancipation, and the widespread isolation of the individual - with an imagination and social agency that conventional decision-making in housing lacked.

These latter examples involved a reform of the practice of the architectural profession becoming infrastructuring ${ }^{35}$ experience of temporal, contingent and social occupations of space. Recent moves of the participatory design field towards 'design-after-design' to design for a continuous appropriation and redesign have summed it up as a move from designing 'things' (objects) to designing 'Things' (socio-material assemblies) ${ }^{36}$. 
Maybe these other experiments of the Nordic countries which could not assign itself any lesser task than 'a world of its own design'37, could help this twenty-first century generation to achieve the revived old goal of a transformative participation.

\section{CONCLUSION}

There is a contemporary constellation of practices, publications, exhibitions and events of architecture with participatory and collective approaches, revealing an undercurrent of present-day society with implications for the housing industry. A critical review of the missteps of the previous generation of architects concerned with the democratisation of the discipline and the profession in the 1960s and 1970s and their architecture of participation reveals the many internal and external problems it faced.

However, experience shows that it was not their demand for collective freedom, but its evolution into struggles for individual autonomy and the resulting withdrawal into illusions of disciplinary autonomy and philosophies of inaction that was the reason for its limited success.

The theory of social construction of technology helps to identify what problems participatory architecture faced with the main stakeholders in the emergence and development of these technological devices. Experience like Flexibo, where the constellation of actors and conditions was optimal, point to a crucial flaw: those efforts towards democratisation of everyday life by architecture came unaccompanied by parallel efforts towards democratisation of the 'everydayness' of architecture: the architect's role and his and hers creative processes.

Rather than reiterating the unsuccessful tactics of the previous era, a de-naturalisation of the 'everydayness' of the architectural profession through an infrastructuring of architects and tenants' practices of housing could resume the increasingly popular old goals of integrating processes of mutual learning and fostering democratic practices in the everyday life.

1. Gesamtkunstwerk, term attributed to the composer Richard Wagner, alludes in architecture to a composition in which all parts have been designed to complement each other in a total work of art.

2. Democracy refers here not to as a good-in-itself, but, as Simone Weil draws on Rousseau, a means towards a good end, 'not that something is right because the people like it, but that, under certain conditions, the will of the people is more likely than any other of being in accordance with justice'. WEIL, Simone. Nota sobre la supresión de los partidos políticos. In: WEIL, Simone; VALLS, Sylvia María (ed.). Profesión de fe. Antología y crítica alrededor de su obra. Versión corregida y aumentada. México: Pleroma, 2007 (1 ${ }^{\text {st }}$ ed. 1957), p. 61-73 (62-63). Weil adds: 'the simple statement of these conditions shows that we have never known anything even remotely resembling a democracy'. Ibid. 64 (both author's translation).

3. This term of Aristotelian origin is used here as adapted to the architectural discourse by Inge Mette Kirkeby and Bent Flyvbjerg: KIRKEBY, Inge Mette Knowledge in the making. In: Architectural Research Quarterly, 13, 2009, p. 307-13. FLYVBJERG, Bent. Phronetic Planning Research: Theoretical and Methodological Reflections. In: Planning Theory and Practice, 5:3, 2004, p. 283-306.

4. For example in the study of urban planning in Barcelona: AIBAR, Eduardo; BIJKER, Wiebe E. Constructing the city: The Cerdá Plan for the extension of Barcelona. In: Science, Technology and Human Values, 22: 1, 1997, p. 3-30.

5. Co-creation alludes generically to any act of collective creation.

6. ARNSTEIN, Sherry R. The ladder of citizen participation. In: Journal of the Institute of American Planners, 34: 4, 1969, p. $216-24$.

7. Relevant literature was accessed during a research stay in an international exchange programme by the first author at the Danish Building Research Institute / Aalborg University.

8. DE CARLO, Giancarlo. Architecture's public. In: BLUNDELL JONES, Peter; PETRESCU, Doina M., TILL, Jeremy, eds.. Architecture and participation. Abingdon: Spon Press, 2005, p. 3-22 (15). First ed.: DE CARLO, Giancarlo de Carlo. II publico dell'architettura. In: Parametro, 5 (1970), p. 4-12.

9. During the 1960s, the word participation had become part of the popular political vocabulary, notably among students. In this context, participation refers to direct democracy as a form of resistance to elitist, and often non-democratically elected, forms of governance, particularly against liberal democratic ethos, and a rediscovery of traditional democratic theory, including the views of Rousseau and Aristotle.

10. CRAWFORD, Margaret. Can architects be socially responsible? In: GHIRARDO, Diane, ed. Out of site: a social criticism of architecture. Seattle: Bay Pres, 1991, p. 27-45 (39).

11. In the fields of philosophy and sociology, agency is defined as the capacity of an agent (a person or another identity) to act in a world.

12. KAMINER, Tahl. Architecture, crisis and resuscitation. New York: Routledge, 2011.

13. QUERRAIN, Anne. How inhabitants can become collective developers: France 1968-2000. In: BLUNDELL JONES, Peter; PETRESCU, Doina M.; TILL, Jeremy, eds. Architecture and participation. Abingdon: Spon Press, 2005, p. 105-15 (113). ELLIN, Nan. Participatory Architecture of the Parisian periphery: Lucien Kroll's Vignes Blanches. Journal of Architectural Education, Routledge, primavera 2000, vol. 53, nº 3, pp. 178-83 (p. 181).

14. Architectural movement from the 70 s and 80 s originated in the United Kingdom that advocated mainly for the involvement of the user in the design, construction and management of the physical environment.

15. HARVEY, David. The New Urbanism and the communitarian trap. In: Harvard Design Magazine [online]. Cambridge (MA): Harvard University Graduate School of Design, 1997, n 1 [accesed: 19-03-2018].

16. CRAWFORD, Margaret. Ibid. p. 43.

17. Everyday life refers here to the theoretical term associated with the writings of Marx about alienation, which was also central to many highly influential at the time anarchist and Marxist scholars, e.g. Colin Ward, Georg Lúkacs, Henri Lefebvre or Michel de Certeau.

18. BLUNDELL JONES, Peter. Sixty-eight and after. In: BLUNDELL JONES, Peter; PETRESCU, Doina M.; TILL, Jeremy, eds. Architecture and participation. Abingdon: Spon Press, 2005, p. 127-40 (134).

19. BOOKCHIN, Murray. Social anarchism or lifestyle anarchism. An unbridgeable chasm. Edinburgh: AK Press, 1995, p. 12-13.

20. HABRAKEN, Nicholas J. Supports: an alternative to mass housing. London: Urban International Press, 1999 (1 $1^{\text {st }}$ ed. 1961).

21. SCHNEIDER, Tatjiana; TILL, Jeremy. Flexible housing. Oxford: Architectural Press, 2007. 
22. Assisted self-help in housing means housing built by families for their own use, all supported by the state. For the history of these models: HARRIS, Richard. Slipping Through the Cracks: The Origins of Aided Self-help Housing, 1918-53. En: Housing Studies, Londres: Carfax Publishing Ltd. 1999, vol. 14, nº 3, pp. $281-309$. 23. MANNICHE, Peter. Living democracy in Denmark: independent farmers, farmer's cooperation, the folk high schools, cooperation in towns, social and cultural activities, social legislation, a Danish village. Copenhagen: G.E.C. Gad Pub., 1970 (1 ${ }^{\text {st }}$ ed. 1952).

24. JENSEN, Lotte; KIRKEGAARD, Ole; PEDERSEN, Dan Ove. Beboerdemokrati og forvaltning i den almene boligsektor. SBi-rapport 332. Copenhagen: SBi, 1999.

25. The name in itself refers to democratic principles (in Danish "fælles" means common).

26. PAGH, Jesper. Tyge Arnfred Interview. In: Arkitektur DK, 02, 2013, p. 58-63 (60).

27. ØRUM-NIELSEN, Jørn. Dwelling: at home, in community, on earth: the significance of tradition in contemporary housing. Copenhagen: Architectural Press, 1996, p. 204

28. ARKITEKTEN. Fleksible boliger, Byggesystem for fleksible boliger i tætte, lave, bebyggelser, udviklet for KAB. In: Arkitekten, 25, 1971, p. 537-545.

29. HOLMBERG, Hartvig, ed. -indret selv Deres bolig. Copenhagen: KAB, 1979.

30. KAB Boligsøgende (Social housing search engine), KAB (Copenhagen Social Housing Association) [accessed 15-09-2015] Available at: https://www.kab-bolig.dk/ boligsoegende/soeg-bolig/afdelingsvisning/afdeling-flexibo.aspx?PID=62149\&View=Apartments

31. TILL, Jeremy. The negotiation of hope. In: BLUNDELL JONES, Peter; PETRESCU, Doina M.; TILL, Jeremy, eds. Architecture and participation. Abingdon: Spon Press, 2005, p. 23-41 (25).

32. ROSE, Gillian. Athens and Jerusalem: A tale of two cities. In: Social and legal studies, 3, 1994, p. 333-48 (336).

33. DE CARLO, Giancarlo. Ibid. p. 15.

34. Co-housing is a community of private houses complemented by extensive community spaces. It is planned and managed by its residents, who are usually people who seek greater interaction with their neighbors.

35. Infrastructuring is a term from participatory design theory associated with Stan Allen's theoretical work. In this context, Infrastructuring must be understood as a prolonged process of technical collaboration in the creation of a system, beyond its limitation to the processes of the design phase, as in the cases of double scale. BJÖGVINSSON, Erling; EHN, Pelle; HILLGREN, Per-Anders. Design Things and Design Thinking: Contemporary Participatory Design Challenges. In: Design Issues, Cambridge (MA): MIT Press, summer 2012, vol. 28, n. 3, pp. 101-16.

36. Ibid. p. 102. Drawing on Bruno Latour writings about the etymology of the English word "thing" as assembly in the ancient Nordic and Germanic societies. 37. DEBORD, Guy. The society of the spectacle. London: Bureau of Public Secrets, 2014 (1 (st $^{\text {ed. }}$ 1967), p. 63. 


\section{Autor imagen y fuente bibliográfica de procedencia}

Información facilitada por los autores de los artículos:

página 18, 1 (Crédito fotográfico gentileza de Federico Cairoli); página 19, 2 y página 20, 3 (Crédito fotográfico José Luis Uribe Ortiz); página 21, 4 (Crédito fotográfico gentileza de Berenice Gómez Crosa), 5 (Crédito boceto detalle constructivo gentileza de Luis Elgué); página 22, 6 (Crédito fotográfico gentileza de Estudio Elgué), 7 (Crédito boceto detalle constructivo gentileza de Lukas Fuster); página 23, 8 y 9 (Crédito fotográfico gentileza de Federico Cairoli), 10 (Crédito boceto detalle constructivo gentileza de José Cubilla); página 24, 11 y 12 (Crédito fotográfico gentileza de Federico Cairoli); página 29, 1 (Biblioteca Nacional de Australia. P490/7. 1918); página 30, 2, página 31, 3 y página 32, 4 (Javier Mosquera González); página 33, 5 (Biblioteca Nacional de Australia. PIC/9929/2029 LOC Cold Store PIC NICH), 6 (Biblioteca Nacional de Australia. PIC/9929/2110); página 34, 7 (Javier Mosquera González); página 35, 8 (Biblioteca Nacional de Australia. PIC/P2145); página 36, 9 (WikimediaCommons. Autor: Martyman); página 38, 10 (Javier Mosquera González); página 45, 1 (Elaboración propia); página 49, 2 (Elaboración propia, basado en información contenida en: MANNICHE, Peter, Living democracy in Denmark: independent farmers, farmer's cooperation, the folk high schools, cooperation in towns, social and cultural activities, social legislation, a Danish village. 2. ed. Copenhagen: G.E.C. Gad Pub., 1970. ISBN 0837139856), 3 (Elaboración propia, basado en la información contenida en: RUONAVAARA, Hannu. Home ownership and the Nordic housing policies in the 'Retrenchment phase'. En: Conference Building on Housing Ownership, Delft 2008; y en: TSENKOVA, Sasha; VESTERGAARD, Hedvig. Social Housing Provision in Copenhagen. Artículo presentado en ENHR 2011, Toulousse, France); página 50, 4 (Elaboración propia, a partir de la documentación original del proyecto aprobada por las autoridades de Copenhague, En base a la documentación cedida por Fællestegnestuen), 5 (Elaboración propia); página 51, 6 y 7 (Documentación cedida por Fællestegnestuen), 8 (HOLMBERG, Hartvig, ed. -indret selv Deres bolig. Copenhague: KAB, 1979, pp. 8-9); página 52, 9 (Documentación cedida por Fællestegnestuen), 10 (HOLMBERG, Hartvig, ed. -indret selv Deres bolig. Copenhague: KAB, 1979, portada y p. 43); página 53, 11 (HOLMBERG, Hartvig, ed. -indret selv Deres bolig. Copenhagen: KAB, 1979, p. 26), 12 (Elaboración propia); página 59, 1 (Parte superior: The American Woman's Home or Principles of Domestic Science Being a Guide to the Formation and Maintenance of Economical Healthful Beautiful and Christian Homes. New York: J. B. Ford and Company, 1869; The New Housekeeping: Efficiency Studies in Home Management. Garden City-New York: Doubleday, Page \& Company, 1913; The Psychology of Management: The Function of the Mind in Determining, Teaching and Installing Methods of Least Waste. New York: Sturgis \& Walton Company, 1914. Parte inferior: Catharine Beecher (Wikipedia Commons), Christine Frederick (Christine Frederick Archive, Schlesinger Library, Radcliffe Institute, Harvard University), Lillian Gilbreth (Lillian Moller Gilbreth Papers, Sophia Smith Collection, Northampton)); página 60, 2 y página 61, 3 (The American Woman's Home or Principles of Domestic Science Being a Guide to the Formation and Maintenance of Economical Healthful Beautiful and Christian Homes. New York: J. B. Ford and Company, 1869, p. 26, 37, 40); página 62, 4 (Christine Frederick Archive, Schlesinger Library, Radcliffe Institute, Harvard University); página 63, 5 (FREDERICK, Christine. The New Housekeeping: Efficiency Studies in Home Management. Garden City-New York: Doubleday, Page \& Company, 1913, p. 53); página 64, 6 (FREDERICK, Christine. Household Engineering: Scientific Management in the Home. Chicago: American School of Home Economics, 1920); página 65, 7 (Kheel Center for Labor-Management Documentation and Archives, Cornell University Library, Management Engineering), 8 (National Museum of American History, Behring Center, Division of Work and Industry Collection); página 66, 9 y 10 (Elaboración realizada por Carmen Espegel); página 67, 11 (Izquierda: BEECHER, Catharine y BEECHER STOWE, Harriet. The American Woman's Home or Principles of Domestic Science Being a Guide to the Formation and Maintenance of Economical Healthful Beautiful and Christian Homes. New York: J. B. Ford and Company, 1869, p. 23. Derecha: Siedlung Römerstad 1927-1928. Das Neue Frankfurt, n.4-5, Abril-Mayo, 1930, p. 76); página 68, 12 (Izquierda: HAYDEN, Dolores. The Grand Domestic Revolution: A History of Feminist Designs for American Homes, Neighborhoods, and Cities. Cambridge (Massachusetts): MIT Press, 1981, p. 30. Derecha: Ernst May und das Neue Frankfurt, 1925-1930. Catálogo de la exposición en el Deutsches Architekturmuseum Frankfurt am Main. Berlin: Wilhelm Ernst \& Sohn Verlag, 1986, p. 153); página 69, 13 (Superior izquierda y derecha: Restitución gráfica realizada por Carmen Espegel. Inferior izquierda: BEECHER, Catharine y BEECHER STOWE, Harriet. The American Woman's Home or Principles of Domestic Science Being a Guide to the Formation and Maintenance of Economical Healthful Beautiful and Christian Homes. New York: J. B. Ford and Company, 1869, pp. 28-30. Inferior derecha: Das Neue Frankfurt, n.6, Junio, 1929, p. 128), 14 (Izquierda: FREDERICK, Christine. Household Engineering: Scientific Management in the Home. Chicago: American School of Home Economics, 1920, p. 32. Derecha: Margarete SchütteLihotzky: Frankfurter Küche, 1926. Colección y Archivo de Margarete Schütte-Lihotzky en la Universidad de Artes Aplicadas de Viena); página 70, 15 (Izquierda: Original films of Frank and Lillian Gilbreth, promovida por Chicago Chapter, Society for the Advancement of Management, 1910-1924. San Francisco: National Film Preservation Foundation. Derecha: Neues Bauen in Frankfurt am Main, dirigida por Paul Wolff. Frankfurt: Wolff-Film, 1928); página 75, 1 (Enrique Jesús Fernández-Vivancos González a partir del plano de Tapiola de Aarne Ervi de 1963); página 76, 2 (Le Carré Bleu. 1960 nº 3); página 77, 3 y 4 (Museum of Finnish Architecture [MFA]); página 78, 5 (Le Carré Bleu.1958 nº 1 pp. 2-3. Fotógrafo: PIETINEN, Otson); página 79, 6 y 7, página 80, 8, 9 y 10 y página 81, 11 y 12 (Museum of Finnish Architecture [MFA]); página 82, 13 (Museum of Finnish Architecture [MFA]. Fotógrafo: LEHTONEN, Kai R); página 83, 14 (Museum of Finnish Architecture [MFA]); página 84, 15 (PIETILÄ, Reima. Noción Imagen Idea. Espoo: Teknillisen Korkeakoulun Ylioppilaskunta. 1975. Lección 5 y Lección 16); página 90, 1 (Natalia Matesanz Ventura); página 93, 2 y 3 (Fotograma del documental dirigido por Christina Holmes. 2015 [en línea] https://www.youtube.com/watch?v=bB-3Bp2pWh8); página 103, 1 (https://lebbeuswoods.files.wordpress.com/2011/06/Iw-mugshot1.jpg [en línea] [consulta: 22-08-2017]. Disponible en: https://lebbeuswoods.wordpress.com/2011/06/29/anti-journey-to-architecture-1/), 2 (https://lebbeuswoods.wordpress. com/2009/10/06/notebook-01-3-the-last/); página 106, 3 (https://lebbeuswoods.files.wordpress.com/2012/01/lbw-411.jpg[en línea] [consulta: 22-08-2017]. Disponible en: https://lebbeuswoods.wordpress.com/2012/01/02/origins/); página 108, 4 (https://lebbeuswoods.wordpress.com/2009/09/27/the-vagrant-light-of-stars/ [consulta: 22-08-2017]), 5 (https://lebbeuswoods.files.wordpress.com/2010/11/dwg-1.jpg [en línea] [consulta: 22-08-2017]. Disponible en: https://lebbeuswoods.wordpress. com/2010/11/02/drawings-stories/); página 109, 6 (https://lebbeuswoods.wordpress.com/2009/08/01/meta-institutes/ [consulta: 22-08-2017]); página 110, 7 (https:// lebbeuswoods.wordpress.com/2009/03/15/notebook-97-3/ [consulta: 22-08-2017]), 8 (https://lebbeuswoods.wordpress.com/2009/12/19/storm-watch/ [consulta: 2208-2017]); página 111, 9 (https://lebbeuswoods.wordpress.com/2009/06/05/architecture-of-energy/ [consulta: 22-08-2017]), 10 (https://lebbeuswoods.wordpress. com/2011/02/15/a-space-of-light-2/ [consulta: 22-08-2017]); página 112, 11 (https://lebbeuswoods.files.wordpress.com/2012/03/Itpav-may-30-3.jpg [en línea] [consulta: 22-08-2017]. Disponible en: https://lebbeuswoods.wordpress.com/2012/03/25/light-pavilion-under-construction/); página 113, 12 (https://lebbeuswoods.files.wordpress. com/2011/01/cdusk1day.jpg [en línea] [consulta: 22-08-2017] Disponible en: https://lebbeuswoods.wordpress.com/2011/02/15/a-space-of-light-2/); página 114, 13 (https://lebbeuswoods.wordpress.com/2009/09/19/line-up/ [consulta: 22-08-2017]), 14 (https://lebbeuswoods.files.wordpress.com/2011/02/int-5-11.jpg [en línea] [consulta: 22-08-2017]. Disponible en: https://lebbeuswoods.wordpress.com/2011/02/15/a-space-of-light-2/), 15 (Fotografía: Shu He. Imagen cedida por Steven Holl Architects) 